\title{
Folding PDZ2 domain using the Molecular Transfer Model
}

\author{
Zhenxing $\mathrm{Liu}^{1}$, Govardhan Reddy ${ }^{2}$, and D. Thirumalai ${ }^{3,4}$ \\ ${ }^{1}$ Department of Physics, Beijing Normal University, Beijing, China 100875 \\ ${ }^{2}$ Solid State and Structural Chemistry Unit, \\ Indian Institute of Science, Bangalore, Karnataka, India 560012 \\ ${ }^{3}$ Biophysics Program, Institute For Physical Science and Technology, \\ University of Maryland, College Park, Maryland 20742 \\ ${ }^{4}$ Department of Chemistry and Biochemistry, \\ University of Maryland, College Park, Maryland 20742
}

(Dated: January 11, 2016) 


\begin{abstract}
A major challenge in molecular simulations is to describe denaturant-dependent folding of proteins order to make direct comparisons with in vitro experiments. We use the molecular transfer model (MTM), which is currently the only method that accomplishes this goal albeit phenomenologically, to quantitatively describe urea-dependent folding of PDZ domain, which plays a significant role in molecular recognition and signaling. Experiments show that urea-dependent unfolding rates of the PDZ2 domain exhibit a downward curvature at high urea concentrations $([C] \mathrm{s})$, which has been interpreted by invoking the presence of a sparsely populated high energy intermediate. Simulations using the MTM and a coarse-grained Self-Organized Polymer (SOP) representation of PDZ2 are used to show that the intermediate $\left(I_{E Q}\right)$, which has some native-like character, is present in equilibrium both in the presence and absence of urea. The free energy profiles as a function of the structural overlap order parameter show that there are two barriers separating the folded and unfolded states. Structures of the transition state ensembles, (TSE1 separating the unfolded and $I_{E Q}$ and TSE2 separating $I_{E Q}$ and the native state), determined using the $P_{\text {fold }}$ method, show that TSE1 is greatly expanded while TSE2 is compact and native-like. Folding trajectories reveal that PDZ2 folds by parallel routes. In one pathway folding occurs exclusively through $I_{1}$, which resembles $I_{E Q}$. In a fraction of trajectories, constituting the second pathway, folding occurs through a combination of $I_{1}$ and a kinetic intermediate. We establish that the radius of gyration $\left(R_{g}^{U}\right)$ of the unfolded state is more compact (by $\sim 9 \%$ ) under native conditions. Theory and simulations show that the decrease in $R_{g}^{U}$ occurs on the time scale on the order of utmost $\sim 20 \mu \mathrm{s}$. The modest decrease in $R_{g}^{U}$ and the rapid collapse suggest that high spatial and temporal resolution, currently beyond the scope of most small angle X-ray scattering experiments, are needed to detect compaction in finite-sized proteins. The present work further establishes that MTM is efficacious in producing nearly quantitative predictions for folding of proteins under conditions used to carry out experiments.
\end{abstract}




\section{Introduction}

The Molecular Transfer Model (MTM) ${ }^{1}$, based on the statistical mechanical theory of liquid mixtures $^{2}$, is currently the only available computational method that predicts the outcomes of experiments and provides the structural basis of folding as a function of denaturants ${ }^{3,4}$ and $\mathrm{pH}^{5}$. Using the MTM in conjunction with coarse-grained (CG) representation of polypeptide chains we have made quantitative predictions of the folding thermodynamics and kinetics as a function of denaturants for a number of small (protein L, cold shock protein, srcSH3 domain, and Ubiquitin) $)^{1,2,4,6}$, and GFP, a large single domain proteins ${ }^{3}$. Because the effects of denaturants are taken into account naturally within the MTM framework ${ }^{2}$, albeit phenomenologically it has been possible to obtain chevron plots for src SH3 domain producing quantitative agreement with experiments for the slopes of folding and unfolding arms ${ }^{7}$. Although MTM can be be implemented in conjunction with atomically detailed simulations we have so far used CG models for proteins. The virtue of CG models ${ }^{8-11}$ is that they can be used to obtain both the thermodynamic and kinetic properties over a a wide range of external conditions, thus allowing us to compare with experiments directly ${ }^{12}$. These studies illustrate that simulations based on the MTM provide concrete predictions for in vitro experiments enabling us to go beyond generic ideas used to understand protein folding ${ }^{13-23}$. Here, we investigate the folding mechanism of PDZ2 domain using CG simulations within the theoretical framework of the MTM.

PDZ domains are a large family of globular proteins that mediate protein-protein interactions and play an important role in molecular recognition ${ }^{24-26}$. These proteins generally consist of 80-100 amino acids. PDZ2 domain has six $\beta$ strands and two $\alpha$ helices (Figure 1a). The folding mechanism of PDZ2, with 94 residues, has been studied experimentally ${ }^{27}$ using classical chemical kinetics methods. The key findings in these experiments are: (i) In urea-induced equilibrium denaturation experiments, the observed transition is cooperative, which is well described by an apparent two-state model ${ }^{27,28}$. (ii) In a majority of cases proteins that fold thermodynamically in a two-state manner also exhibit a similar behavior kinetically in ensemble experiments. However, urea-dependent unfolding rates exhibit a downward curvature at high urea concentrations at $\mathrm{pH}$ $>$ 5.5. Based on the observation that the folding kinetics is mono-phasic, with no detectable burst phase in the initial fluorescence of the initial unfolding time course, it was surmised that there is no low energy intermediate in the unfolding of PDZ2. Rather the data were used to 
suggest the presence of a high-energy on-pathway intermediate, which does not accumulate significantly in equilibrium ${ }^{27}$. (iii) The high energy intermediate is non-detectable under stabilizing conditions, achievable in PDZ2 domain by addition of modest amount of sodium sulfate. Under these conditions PDZ2 folds thermodynamically and kinetically in a two-state manner. (iv) The structures of the two transition state ensembles were also inferred using measured $\Phi$ values as constraints in all atom molecular dynamics simulations ${ }^{29}$. Unlike the results summarized in (i)(iii) the predicted structures of the transition state ensembles are not as conclusive for reasons explained later in this work.

In order to provide a comprehensive picture of folding of PDZ2, with potential implications for other single domain proteins, we performed molecular simulations of a coarse-grained offlattice model with side chains ${ }^{9,30}$ and used $\mathrm{MTM}^{1,3,7}$ to account for for denaturant effects. The free energy profiles as a function of the structural overlap order parameter at different urea concentrations, $[C]$, and temperature $T$ suggest that the folding mechanism of PDZ2 can be altered by changing the stability of the folded state. In accord with experiments, we demonstrate directly the existence of the fleeting obligatory intermediate both in equilibrium $\left(I_{E Q}\right)$ and kinetics $\left(I_{1}\right)^{31}$. The structures of $I_{E Q}$ and $I_{1}$ are similar. However, the fraction of molecules in intermediate basin of attraction (IBA), $f_{I B A}$, as a function of temperature and $[C]$ is small, thus explaining the difficulty in detecting it in standard denaturation experiments. In addition to $I_{1}$ a kinetic intermediate, $I_{2}$, is consistently populated in $\sim 53 \%$ of the folding trajectories. Guided by the free energy profiles, we identified two transition state ensembles, TSE1 and TSE2. The computed values of the Tanford-like $\beta$ parameters, using the solvent accessible surface area as a surrogate, for the two transition state ensembles (one connecting the NBA and the IBA and the other involving transition between the IBA and the UBA) are in qualitative agreement with those obtained from experiments ${ }^{27,31}$. The current work further establishes that simulations based on the MTM are efficacious in providing a nearly quantitative picture of folding of single domain proteins.

\section{METHODS}

SOP-sidechain model: The simulations were carried out using a CG model in which the $C_{\alpha}$-based self-organized polymer (SOP) representation ${ }^{32}$ was augmented to include side chains 
$(\mathrm{SCs})^{2,7}$. In the SOP-SC model each residue is represented by two interaction centers, one that is located at the $\mathrm{C}_{\alpha}$ position and the other at the center of mass of the side chain. In the SOP-SC model the native state stabilization is achieved by accounting for backbone-backbone (bb), side chain-side chain (ss), and backbone-side chain (bs) interactions present in the folded state. Neglect of non-native interactions, which do not significantly alter the folding mechanism beyond the global collapse of the protein ${ }^{33-36}$, is nevertheless a limitation of the model.

The energy (to be interpreted as an effective free energy obtained by integrating over solvent (water) degrees of freedom) of a conformation, describing the intra peptide interactions, is

$$
E_{P}\left(\left\{r_{i}\right\}\right)=V_{F E N E}+V_{L J}^{N A T}+V^{N E I}+V_{L J}^{N N}
$$

The finite extensible nonlinear elastic potential (FENE), $V_{F E N E}$, accounting for the chain connectivity between backbones and side chains, is,

$$
\begin{aligned}
V_{F E N E}= & V_{F E N E}^{b b}+V_{F E N E}^{b s} \\
= & -\sum_{i=1}^{N-1} \frac{k}{2} R_{o}^{2} \log \left(1-\frac{\left(r_{i, i+1 \_b b}-r_{i, i+1 \_b b}^{o}\right)^{2}}{R_{o}^{2}}\right) \\
& -\sum_{i=1}^{N} \frac{k}{2} R_{o}^{2} \log \left(1-\frac{\left(r_{i, i \_b s}-r_{i, i \_b s}^{o}\right)^{2}}{R_{o}^{2}}\right) .
\end{aligned}
$$

The non-bonded native interaction, $V_{L J}^{N A T}$ in Eq. (1) is taken to be

$$
\begin{aligned}
& V_{L J}^{N A T}=V_{L J_{-} N A T}^{b b}+V_{L J_{-} N A T}^{s s}+V_{L J_{-} N A T}^{b s} \\
& =\sum_{i=1}^{N-3} \sum_{j=i+3}^{N} e_{b b}\left[\left(\frac{r_{i, j_{\_} b b}^{o}}{r_{i, j_{\_} b b}}\right)^{12}-2\left(\frac{r_{i, j_{\_} b b}^{o}}{r_{i, j_{\_} b b}}\right)^{6}\right] \Delta_{i j}^{b b} \\
& +\sum_{i=1}^{N-3} \sum_{j=i+3}^{N} e_{s s}\left|\epsilon_{i j}-0.7\right|\left[\left(\frac{r_{i, j_{-s s}}^{o}}{r_{i, j_{-} s s}}\right)^{12}-2\left(\frac{r_{i, j_{-} s s}^{o}}{r_{i, j_{-} s}}\right)^{6}\right] \Delta_{i j}^{s s} \\
& +\sum_{\substack{i=1, j=1 \\
|i-j| \geq 3}}^{N} e_{b s}\left[\left(\frac{r_{i, j_{\_} b s}^{o}}{r_{i, j \_b s}}\right)^{12}-2\left(\frac{r_{i, j_{\_} b s}^{o}}{r_{i, j \_b s}}\right)^{6}\right] \Delta_{i j}^{b s} \text {. }
\end{aligned}
$$

In Eqs. 2 and 3 the superscript ${ }^{o}$ refers to distances in the native state. If the distance between two non-covalently linked beads, $r_{i j}(|i-j| \geq 3)$ in the PDB structure is within a cutoff distance $R_{c}$, a native contact is formed, and correspondingly $\Delta_{i j}=1$. If $r_{i j}$ exceeds $R_{c}$ then $\Delta_{i j}=0$. The strengths of the non-bonded interactions $e_{b b}, e_{s s}, e_{b s}$ are assumed to be uniform. 
The Betancourt-Thirumalai $(\mathrm{BT})^{37}$ statistical potential matrix with elements $\epsilon_{i j}$, is used to explicitly treat the sequence dependence.

We used repulsive interactions for excluded volume effects between neighboring beads with strength $e_{l}$. The ranges of repulsion are $\sigma_{b b}, \sigma_{i, j_{-} s s}, \sigma_{j_{-} b s}$ for bb, ss and bs interactions respectively. The form of $V^{N E I}$ is

$$
\begin{aligned}
V^{N E I} & =V_{N E I}^{b b}+V_{N E I}^{s s}+V_{N E I}^{b s} \\
& =\sum_{i=1}^{N-2} e_{l}\left(\frac{\sigma_{b b}}{r_{i, i+2 \_b b}}\right)^{6} \\
& +\sum_{i=1}^{N-1} e_{l}\left(\frac{\sigma_{i, i+1 \_s s}}{r_{i, i+1 \_s s}}\right)^{6}+\sum_{i=1}^{N-2} e_{l}\left(\frac{\sigma_{i, i+2 \_s s}}{r_{i, i+2 \_s s}}\right)^{6} \\
& +\sum_{\substack{i=1, j=1 \\
0<|i-j|<3}}^{N} e_{l}\left(\frac{\sigma_{j \_b s}}{r_{i, j \_b s}}\right)^{6} .
\end{aligned}
$$

To prevent interchain crossing, we choose $\sigma_{b b}=a=3.8 \AA$ ( $a$ is average distance between neighboring $C_{\alpha}$ atoms $), \sigma_{i, j_{-} s s}=f\left(\sigma_{i}+\sigma_{j}\right)\left(\sigma_{i}, \sigma_{j}\right.$ are the van der Waals radii of the side chains and $f=0.5), \sigma_{j_{-} b s}=f\left(a+\sigma_{j}\right)$.

The non-bonded nonnative interactions are given by

$$
\begin{aligned}
V_{L J}^{N N} & =V_{L J_{-} N N}^{b b}+V_{L J_{-N N}}^{s s}+V_{L J_{-N N}}^{b s} \\
& =\sum_{i=1}^{N-3} \sum_{j=i+3}^{N} e_{l}\left(\frac{\sigma_{b b}}{r_{i, j_{\_} b b}}\right)^{6}\left(1-\Delta_{i j}^{b b}\right) \\
& +\sum_{i=1}^{N-3} \sum_{j=i+3}^{N} e_{l}\left(\frac{\sigma_{i, j \_s s}}{r_{i, j \_s s}}\right)^{6}\left(1-\Delta_{i j}^{s s}\right) \\
& +\sum_{\substack{i=1, j=1 \\
|i-j| \geq 3}}^{N} e_{l}\left(\frac{\sigma_{j_{\_} b s}}{r_{i, j \_b s}}\right)^{6}\left(1-\Delta_{i j}^{b s}\right) .
\end{aligned}
$$

Besides the knowledge-based BT statistical potential, the SOP-SC energy function $E_{P}\left(\left\{r_{i}\right\}\right)$ has seven parameters: $R_{o}=2 \AA, k=20 \mathrm{kcal} /\left(\mathrm{mol} \cdot \stackrel{\AA}{A}^{2}\right), R_{c}=8 \AA, e_{b b}=0.73 \mathrm{kcal} / \mathrm{mol}, e_{b s}=$ $0.17 \mathrm{kcal} / \mathrm{mol}, e_{s s}=0.3 \mathrm{kcal} / \mathrm{mol}, e_{l}=1 \mathrm{kcal} / \mathrm{mol}$. Among these parameters, $R_{o}$ and $k$ merely account for chain connectivity. The results would not be significantly affected by the precise choice of parameters enforcing the integrity of the polypeptide chain. Thus, in effect there are five parameters in the SOP-SC model. Analysis of PDB structures shows that $R_{c}=8 \AA$ 
is a reasonable choice. By analyzing structures of folded proteins in the Protein Data Bank (PDB) that there are large favorable bb and bs contacts ${ }^{38}$. The first and third terms account for these interactions, which in turn ensures that packing effects are appropriately described in the SOP-SC model. The experimental melting temperature of the protein is used to determine the strengths of the native contacts, specified by $e_{b b}, e_{b s}, e_{s s}$.

Molecular Transfer Model: The MTM theory ${ }^{2}$ shows that experimentally measured transfer free energies for backbone and side chains along with the SOP-SC model could be utilized to obtain the partition function for a protein at a finite denaturant concentration $[\mathrm{C}]^{2}$. In the MTM, the free energy of transferring a given protein conformation, described by $\left\{r_{i}\right\}$ from water $([\mathrm{C}]=0)$ to aqueous denaturant solution $([\mathrm{C}] \neq 0)$, is approximated as,

$$
\Delta G\left(\left\{r_{i}\right\},[C]\right)=\sum_{i} \delta g(i,[C]) \alpha_{i} / \alpha_{G l y-i-G l y}
$$

where the sum is over backbone and side chain, $\delta g(i,[C])$ is the experimentally measured transfer free energy of group $i, \alpha_{i}$ is the solvent accessible surface area (SASA), and $\alpha_{G l y-i-G l y}$ is the SASA of the $i^{t h}$ group in the tripeptide Gly $-i-G l y$. Thus, the effective free energy function for a protein at $[\mathrm{C}] \neq 0$ is

$$
H_{P}\left(\left\{r_{i}\right\},[C]\right)=E_{P}\left(\left\{r_{i}\right\}\right)+\Delta G\left(\left\{r_{i}\right\},[C]\right)
$$

For computational expediency we combined converged simulations at finite temperature using $E_{P}\left(\left\{r_{i}\right\}\right)$ and computed the partition function at $[\mathrm{C}] \neq 0$ to obtain thermodynamic properties using a weighted histogram analysis method, which takes into account the effects of $\Delta G\left(\left\{r_{i}\right\},[C]\right)$. Such an approximation, whose validity for obtaining thermodynamic properties has been previously established ${ }^{7}$, is used to obtain thermodynamic properties.

Langevin and Brownian Dynamics Simulations: We assume that the dynamics of the protein is governed by the Langevin equation, which includes a damping term with a friction coefficient $\zeta$, and a Gaussian random force $\Gamma$. The equation of motion for a generalized coordinate $r_{i}$ is $m \ddot{\mathrm{r}}_{i}=-\zeta \dot{\mathrm{r}}_{i}+F_{c}+\Gamma$ where $m$ is the mass of a bead, $F_{c}=-\partial E_{P}\left(\left\{r_{i}\right\}\right) / \partial r_{i}$, is the conformational force calculated using Eq. (1), $\Gamma$ is the random force with a white noise spectrum. The autocorrelation function for $\Gamma(t)$ in the discretized form is $\left\langle\Gamma(t) \Gamma(t+n h)>=\frac{2 \zeta k_{B} T}{h} \delta_{0, n}{ }^{39}\right.$ where $\delta_{0, n}$ is the Kronecker delta function and $n=0,1,2 \ldots$ The value of the time step, $h$, depends on the friction coefficient $\zeta$. 
To obtain enhanced sampling, we used the Replica Exchange Molecular Dynamics $(\mathrm{REMD})^{40-43}$ to perform thermodynamics sampling using a low friction coefficient $\zeta=$ $0.05 \mathrm{~m} / \tau_{L}{ }^{44}$, which allows us to accurately calculate the equilibrium properties. Rapid convergence of thermodynamics is possible at a small $\zeta$ (under damped limit) because the polypeptide chain makes frequent transitions between all accessible states. In the low $\zeta$ limit limit, we used the Verlet leap-frog algorithm to integrate the equations of motion. The velocity at time $t+h / 2$ and the position at time $t+h$ of a bead are given by,

$$
\begin{aligned}
v_{i}(t+h / 2) & =\frac{2 m-h \zeta}{2 m+h \zeta} \cdot v_{i}(t-h / 2)+\frac{2 h}{2 m+h \zeta}\left[F_{c}(t)+\Gamma(t)\right] \\
r_{i}(t+h) & =r_{i}(t)+h \cdot v_{i}(t+h / 2)
\end{aligned}
$$

In order to simulate the kinetics of folding, $\zeta$ is set to be $50 \mathrm{~m} / \tau_{L}$, which approximately corresponds to the value in water and represents the over damped $\operatorname{limit}^{39}$. In the high $\zeta$ value, we use the Brownian dynamics algorithm ${ }^{45}$, which allows us to integrate equations of motion using

$$
r_{i}(t+h)=r_{i}(t)+\frac{h}{\zeta}\left(F_{c}(t)+\Gamma(t)\right)
$$

Time Scales: The natural unit of time for over damped condition at the transition temperature $T_{s}$ is $\tau_{H} \approx \frac{\zeta_{H} a^{2}}{k_{B} T_{s}}=\frac{\left(\zeta_{H} \tau_{L} / m\right) e_{l}}{k_{B} T_{s}} \tau_{L}$. To convert the simulation time to real time, we chose $e_{l}=1 \mathrm{kcal} / \mathrm{mol}$, average mass $m=1.8 \times 10^{-22} \mathrm{~g}^{39}, a=4 \stackrel{\circ}{A}$, which makes $\tau_{L}=2 p s$. For $\zeta_{H}=50 \mathrm{~m} / \tau_{L}$, we obtain $\tau_{H}=159 \mathrm{ps}$. For thermal folding simulations, the integration time step, $h$ is $0.005 \tau_{L}$. In the kinetic folding simulations, $h$, in Eq.(10) is $0.02 \tau_{H}$.

Data Analysis: The [C]-dependent melting temperature is identified with the peak in the heat capacity. The structural overlap function $\chi=1-\frac{N}{k}_{T} 46$ is used to monitor the folding reaction, where

$$
\begin{aligned}
N_{k} & =\sum_{i=1}^{N-3} \sum_{j=i+3}^{N} \Theta\left(\delta-\left|r_{i, j_{\_} b b}-r_{i, j_{\_} b b}^{o}\right|\right)+\sum_{i=1}^{N-3} \sum_{j=i+3}^{N} \Theta\left(\delta-\left|r_{i, j_{\_} s s}-r_{i, j_{\_} s s}^{o}\right|\right) \\
& +\sum_{\substack{i=1, j=1 \\
|i-j| \geq 3}}^{N} \Theta\left(\delta-\left|r_{i, j_{\_} b s}-r_{i, j \_b s}^{o}\right|\right)
\end{aligned}
$$

In Eq.(11), $\Theta(x)$ is the Heavyside function. If $\left|r_{i, j}-r_{i, j}^{o}\right| \leq \delta(=2 \AA)$, there is a contact. The number of contacts in the $k^{t h}$ conformation is $N_{k}$, and $N_{T}$ is the total number in the folded state. 


\section{Results}

Thermal Denaturation:: The temperature dependence of the heat capacity, $C_{v}(=$ $\frac{\left.\leq E^{2}>-<E\right\rangle^{2}}{k_{B} T^{2}}$ ), where $\left\langle E>\right.$ and $\left\langle E^{2}>\right.$ are the mean and mean square averages of the energy, respectively, demonstrates that PDZ2 folds cooperatively in a two-state manner (Figure 1b). The melting temperature, identified with the peak in $C_{v}(T)$, is $T_{m}=324 K$. The value of $T_{m}$ obtained in our simulations is in excellent agreement with the experimentally measured $T_{m}=321 K^{47}$.

In order to identify the $N B A, U B A$, and the intermediate basin of attraction (IBA) representing the $I_{E Q}$ state, we plot in Figure 1c the free energy $(G(\chi))$ profile as function of $\chi$ at $T_{m}=324 K$. All the conformations can be classified into three states specified by the black vertical lines based on the $\chi$ values. If $\chi \leq \chi_{c}^{N}=0.64$, the conformations are in the NBA, conformations with $\chi \geq \chi_{c}^{D}=0.825$ belong to the UBA, and the rest of the conformations are in the IBA. The fractions of molecules in the $N B A, f_{N B A}([0], T)$ (the first argument shows the value of the denaturant concentration), in the $U B A f_{U B A}([0], T)$, and in the $I B A, f_{I B A}([0], T)$ as a function of temperature are plotted in Figure 1d. Both $f_{N B A}([0], T)$ and $f_{U B A}([0], T)$ show that the folding or unfolding is cooperative. The value of $f_{I B A}([0], T)$ is negligible compared to $f_{N B A}([0], T)$ and $f_{U B A}([0], T)$, suggesting that from a thermodynamic perspective a two-state description is adequate, reflecting the cooperative transition in the heat capacity curve (Figure 1b). The sparse population of the $I B A$ explains why the $I_{E Q}$ state is hard to detect in experiments although its presence appears as a shoulder in the free energy profile (Figure 1c). The value of $T_{m}$ computed using $f_{N B A}\left(T_{m}\right)=0.5$ yields $T_{m}=324 K$, which coincides with the peak in the heat capacity (Figure 1b).

Chemical Denaturation: Following our previous studies ${ }^{1,3,7}$, we choose a simulation temperature, $T_{s}$, at which the calculated free energy difference between the native state $(\mathrm{N})$ and the unfolded state $(\mathrm{U}), \Delta G_{N U}\left(T_{s}\right)\left(G_{N}\left(T_{s}\right)-G_{U}\left(T_{s}\right)\right)$ and the measured free energy $\Delta G_{N U}\left(T_{E}\right)$ at $T_{E}(=298 \mathrm{~K})$ coincide. The use of $\Delta G_{N U}\left(T_{s}\right)=\Delta G_{N U}\left(T_{E}\right)$ ( in water with $[C]=0$ ) to fix $T_{s}$ is equivalent to choosing a overall reference energy scale in the simulations. For PDZ2, $\Delta G_{N U}\left(T_{E}=298 \mathrm{~K}\right)=-3.1 \mathrm{kcal} / \mathrm{mol}$ at $[C]=0^{28}$, which results in $T_{s}=317 \mathrm{~K}$. Besides the choice of $T_{s}$, no other parameter is adjusted to obtain agreement with experiments for any property. 
With $T_{s}=317 K$ fixed, we calculated the dependence of $f_{N B A}\left([C], T_{s}\right), f_{U B A}\left([C], T_{s}\right)$, and $f_{I B A}\left([C], T_{s}\right)$ on $[C]$ (Figure 2a). The agreement between the measured and simulated results for $f_{N B A}\left([C], T_{s}\right)$ as a function of $[\mathrm{C}]$ is excellent (Figure 2a). We find, just as in thermal denaturation, that $f_{I B A}\left([C], T_{s}\right)$ is small ${ }^{27,28}$. The midpoint concentration, $C_{m}$, obtained using $f_{N B A}\left(\left[C_{m}\right], T_{s}\right)=0.5$ is $[\mathrm{C}]=2.3 \mathrm{M}$, agrees well with the experimentally measured value of $2.6 \mathrm{M}$ (see Figure 6D in ${ }^{28}$ ).

The native state stability with respect to $\mathrm{U}, \Delta G_{N U}([C])\left(=G_{N}([C])-G_{U}([C])\right)$, is computed using $\Delta G_{N U}([C])=-k_{B} T_{s} \ln \left(\frac{f_{N B A}}{f_{U B A}}\right)$. The linear fit, $\Delta G_{N U}([C])=\Delta G_{N U}(0)+m[C]$, yields $\Delta G_{N U}([0])=-3.09 \mathrm{kcal} / \mathrm{mol}$ and $m=1.35 \mathrm{kcal} / \mathrm{mol} \cdot M$ (Figure 2b). The experimentally inferred $m=1.20 \mathrm{kcal} / \mathrm{mol} \cdot M$ compares well with the simulations, which establishes again that simulations based on MTM predict the thermodynamic properties of proteins accurately. The $[C]$-dependent heat capacity curves (Figure 2c) show that the peaks corresponding to $T_{m}([C]$ ) decreases as $[C]$ increases (Figure $2 \mathrm{c}=\mathrm{d}$ ). Taken together the simulations show that the equilibrium folding induced by temperature or denaturants is cooperative.

Urea-dependent changes in the shape of PDZ2: The dependence of the radius of gyration, $\left\langle R_{g}([C])\right\rangle$, on urea concentration (black line in Figure 3a) shows a transition from an expanded to a collapsed state as $[C]$ decreases. The radii of gyration of the $I_{E Q}$ and the native state are virtually constant at all urea concentrations. However, the radius of gyration of the $U B A$ decreases as $[C]$ decreases implying that the ensemble of structures of the unfolded state is more compact under native conditions than at $8 \mathrm{M}$ urea. The decrease in the $R_{g}$ of the $U B A$ structures in going from $8 \mathrm{M}$ urea to $1 \mathrm{M}$ is about $9 \%$, which should be measurable in a high precision Small Angle X-ray scattering (SAXS) experiment (see below for additional discussion). The $P\left(R_{g}\right)$ distributions at various urea concentrations show the expected behavior (Figure $3 \mathrm{~b}$ ). The protein is largely in the $N B A$ at $[C]$ less than $2.3 \mathrm{M}$ (mid point of the folding transition) and is expanded at higher values of $[C]$. The distance distributions, which can be measured as the inverse Fourier transform of the wave vector dependent scattering intensity, are plotted in Figure $3 \mathrm{c}$ and constitutes one of the predictions.

Free energy profiles, $G(\chi)$, reveal lowly populated intermediate: To illustrate how urea changes the folding landscape, we plotted $G(\chi)$ versus $\chi$ at different $[C]$ at $T=T_{m}=$ $324 K$ in Figure $4 \mathrm{a}$ and at $T=T_{s}=317 K$ in Figure $4 \mathrm{~b}$. Figure $4 \mathrm{a}$ shows, that at all urea concentrations, the conformations could be partitioned into three states, which is consistent 
with the results in Figure $1 c$ displaying $G(\chi)$ at $T_{m}$ with $[C]=0$. The basin of attraction corresponding to $I_{E Q}$ becomes deeper as $[C]$ increases but is shallow compared to the $N B A$ and $U B A$. The barrier for TSE1 is lower than for TSE2, implying that the transition from the intermediate states to the UBA is more facile than transition to the $N B A$. The number of distinct minima remain unchanged at the lower temperature, $T_{s}=317 \mathrm{~K}$ (Figure $4 \mathrm{~b}$ ). However, the basin for the intermediate states becomes shallower as $[C]$ increases, and the barrier for TSE1 almost disappears, especially when $[C]>C_{m}=2.3 M$, which indicates that the intermediate, if it can be detected at all, is unstable. The absence of $I_{E Q}$ at the lower temperature suggests that stabilization of the native fold of PDZ2 leads to a two-state thermodynamic transition. Our finding supports the same observation in experiments showing that native state stabilization upon addition of sodium sulfate results in the high energy $I_{E Q}$ being undetectable.

Structures of the transition state ensembles (TSE1 and TSE2): We identified the transition state structures using the folding trajectories generated at $T_{m}$. We picked the putative transition state from the barrier regions of the free energy profile as a function of $\chi$, using the conditions $0.815<\chi<0.835$ for TSE1 and $0.63<\chi<0.65$ for TSE2, which are represented as shaded areas in Figure 1c. Starting from these structures, we calculated the commitment probability, $P_{\text {fold }}$, of reaching the $N B A^{48}$. The sets of structures with $0.4 \leqslant P_{\text {fold }} \leqslant 0.6$ are identified as the transition state ensemble. The characteristics of the two transition state ensembles are displayed in Figure 5a and the distribution of the structural order parameter $P(\chi)$ of the TSE1 along with the contact map are shown in Figure 5b and Figure 5c, respectively. Distribution of $P(\chi)$ and the structural details for TSE2 are shown in Figures 5d and 5e, respectively.

The characteristics of the TSEs are experimentally described using the Tanford $\beta$ parameter. By fitting the measured Chevron plots as linear function of $[C]$ it has been shown that $\beta_{E}^{1}=0.53$ for TSE1, and $\beta_{E}^{2}=0.89$ for TSE2 ${ }^{27}$ and $\beta_{E}^{1}=0.35$ for TSE1 and $\beta_{E}^{2}=0.85$ for TSE2 $2^{31}$. It is generally assumed that $\beta$ is related to the buried solvent accessible surface area (SASA) in the transition state. For the TSEs obtained in our simulations, we calculated the distribution $P\left(\Delta_{R}\right)$ (see Figure 5a), where $\Delta_{R}=\left(\Delta_{U}-\Delta_{T S E}\right) /\left(\Delta_{U}-\Delta_{N}\right)$ with $\Delta_{U}, \Delta_{T S E}, \Delta_{N}$ being the SASAs in the DSE $([C]=8.0 M)$, TSE, and the NBA $([C]=0.0 M)$, respectively. We found that the average $\left\langle\Delta_{R}>=0.23=\beta_{s}^{1}\right.$ for TSE1 and $\left\langle\Delta_{R}>=0.68=\beta_{s}^{2}\right.$ for TSE2, which qualitatively agree with the values inferred from experiments. The small value of $\beta_{1}$ suggests 
that the $T S E 1$ structures are more $U B A$-like whereas the TSE2 structures resemble the native state.

The structural details are revealed in Figure $5 \mathrm{c}$, which shows the contact map for TSE1. It is clear that relative to the native state (top half in Figure $5 \mathrm{c}$ ) the extent to which the structure is ordered in TSE 1 is modest (green) to low (blue). The contacts between $\beta$ strands $1-6-4$ have low formation probabilities as indicated by the two black circled regions. The secondary structures, $\beta_{23}$ and two helices, are ordered to a greater extent. A representative structure for $T S E 1$ displayed on the left of Figure 5a shows that the structure is expanded. Only $\beta_{23}$ and the two helices have relatively high formation probability.

The contact map for TSE2 (Figure 5e) shows that the formation probabilities of contacts even between residues that are distant in sequence are high, which results in the ensemble of TSE2 structures being compact. The major blue region in the contact map indicates that $\beta_{16}$ is still largely unstructured. Four superimposed representative structures from TSE2 are shown on the right of Figure 5a. The structures are native-like except that $\beta$ strand 1 is not as well packed as in the native state. The lack of stabilizing interactions in $\beta_{16}$ found in our simulations disagrees with the inferences from the all atom molecular dynamics simulations using measured $\Phi$ values as constraints ${ }^{29}$ (see below for additional discussion).

Folding and collapse kinetics: We calculated the collapse and folding rates at zero urea concentration from the folding trajectories, which were generated from Brownian dynamics simulations $^{39}$. From 93 folding trajectories, the fraction of unfolded molecules at time $t$, is computed using $P_{u}(t)=1-\int_{0}^{t} P_{f p}(s) d s$, where $P_{f p}(s)$ is the distribution of first passage times. We fit $P_{u}(t)=e^{-t k_{f}}$ ( Figure 6a) with $k_{f}=172 s^{-1}$, a value that is about 60 times larger than found in the experiments $\left(2.8^{-1}\right)^{27}$. The kinetics of collapse of PDZ2 domain, shows that $<R_{g}(t)>$ decays with a single rate constant, $k_{c}([C])$, the rate of collapse. The extracted values of $k_{c}([C])=244 s^{-1}$ from the data in Figure $6 \mathrm{~b}$ is greater than $k_{f}([C])$, which shows compaction occurs ahead of folding.

Thermodynamic and kinetic intermediates: Analyses of the folding trajectories show that the folded state is reached through a kinetic intermediate state, $I_{1}$ (Figures 7a, 7b, 7c, and 7d). This intermediate state, while not directly detected in experiment, is likely responsible for the downward curvature observed in the unfolding arm of the chevon $\operatorname{plots}^{27}$. We also find the presence of another kinetic intermediate states $\left(I_{2}\right)$ in some of the trajectories. A quantitative 
analyses allows us to classify the occurrence of $I_{1}$ and $I_{2}$ in the 93 folding trajectories. In 49 trajectories both $I_{1}$ and $I_{2}$ are found. Two trajectories in which this occurs are illustrated in Figures $7 \mathrm{a}$ and $7 \mathrm{~b}$. In Figure $7 \mathrm{a}$, PDZ2 samples $I_{1}$ and $I_{2}$ only once before reaching the native state whereas in Figure 7b, it samples $I_{1}$ and $I_{2}$ more than once. In the second class of trajectories (44 out of 93) PDZ2 samples only the $I_{1}$ (see Figures 7c and 7d for examples). Structures of $I_{1}$ and $I_{2}$ are displayed in Figure 7e.

$I_{E Q}$ and $I_{1}$ are structurally similar: To illustrate the structural similarity between $I_{E Q}$, identified in the free energy profiles (Figures 1 and 4), and $I_{1}$ in $100 \%$ of the kinetic folding trajectories, and $I_{2}$ sampled in some of the folding trajectories, we computed the average fraction of native contacts formed by every residue, $f_{Q}$, for the three states (Figures $8 \mathrm{a}$ and $8 \mathrm{~b}$ ). The correlation between $I_{E Q}$ and $I_{1}$, shown in Figure 8c, is very high $(\mathrm{R}=0.995)$. Therefore, we surmise that $I_{1}$ and $I_{E Q}$ are structurally identical. Quantitative results in Figures $8 \mathrm{~b}$ and $8 \mathrm{~d}$ and sample structures (Figure 8e) show that the structure of $I_{2}$ differs from $I_{E Q}$. Taken together these results show that both thermodynamic and kinetic intermediates can be sampled during the folding process although the former is far more prevalent.

\section{Discussion:}

Minimum Energy Compact Structures (MECS): The folding trajectories reveal that two major intermediates are sampled as PDZ2 folds. The equilibrium intermediate is found in all the trajectories whereas the kinetic intermediate is found in only a fraction of the trajectories. Two aspects of these findings, which are of general validity for folding, are worth pointing out. (i) Both $I_{1}$ and $I_{2}$ form on the time scale of collapse. In those trajectories in which $I_{2}$ forms there frequent transitions between $I_{2}$ and $I_{1}$ (see Figure 7). In the process of making such transitions PDZ2 undergoes considerable expansion. (ii) The intermediates, $I_{E Q}$ and $I_{1}$, are compact and contain native-like features. The major difference between $I_{1}$ and the folded state is in the extent of structure in $\beta_{1}, \beta_{4}$, and $\beta_{6}$ as well as $\alpha_{2}$. The structure of $I_{1}$ is similar to that found in TSE1, which follows from the Hammond postulate. These intermediates, which are like MECS (minimum energy compact structures) ${ }^{49}$ facilitate rapid folding. Although they are difficult to detect in ensemble experiments, single molecule pulling experiments using cycles of force increase and force quench can be used to detect MECS as has been done for Ubiquitin ${ }^{50}$. 
It would be interesting to do similar experiments on PDZ2 to directly observe $I_{1}$ and $I_{2}$ using force as a perturbation.

Collapse and folding transition: Is the size of the polypeptide chain of the unfolded state under folding conditions $\left([C]<C_{m}\right)$ less than at $[C]>C_{m}$ ? Theoretical arguments ${ }^{46,51,52}$, simulations $^{1}$, and a number of single molecule FRET and SAXS experiments ${ }^{19,53}$ have answered the question in the affirmative whereas some SAXS experiments on protein L suggest that there is no evidence for polypeptide chain collapse, which is manifestly unphysical. The arguments in favor of collapse preceding folding is based on the following observations. The random coil state of a polypeptide chain with $N$ residues is expected to be $R_{g}^{U} \sim a_{R} N^{0.6}$ with $a_{R} \approx 2.0 \AA$. This estimate is likely to be an upper bound because even $8 \mathrm{M}$ urea is not a good solvent because even at these elevated concentrations the unfolded state has residual structure. As the denaturant concentration decreases the maximum decrease in $R_{g}$ is likely to have a lower bound $a_{D} N^{0.5}$. It cannot be maximally compact because if it were so then enthalpic interactions would drive these structures to the folded state for which $R_{g} \approx a_{N} N^{1 / 3}$ with $a_{N}=3.0 \AA$ ? . Thus, we surmise that $a_{D} N^{0.5}<R_{g}^{U}<a_{R} N^{0.6}$. The reduction in $R_{g}$ of the unfolded state for $N=64$ (protein L) is predicted to be between (5-10)\% depending on the values of $a_{R}$ and $a_{D}$. In PDZ2 $(N=94)$ we find that the unfolded state $R_{g}$ decreases by only $9 \%$ as $[C]$ decreases (Figure $3 \mathrm{a}$ ). Thus, due to finite $N$ resulting in a small decrease in the unfolded state $R_{g}$ requires high precision SAXS measurements to measure changes in $R_{g}^{U}$ as $[C]$ decreases. The errors in SAXS for protein $\mathrm{L}$ are far too large to accurately estimate $R_{g}^{U}$, especially under native conditions.

The absence of detectable contracted form of the polypeptide chain in time resolved SAXS experiments protein $L$ was used as further evidence that compact states are not formed in the folding of any single domain protein. Using theory ${ }^{54-56}$ it can be shown that collapse of the unfolded state occurs on time scales on the order of $\tau_{c} \approx \tau_{0} N^{\beta}$ where $\beta$ is between 1.5 and 2.2 , and $\tau_{0} \approx \mathcal{O} 10^{-9} s$. For PDZ2 we estimate that $\tau_{c} \sim 20 \mu s$. Our simulations show that the contraction of the unfolded state occurs on time scale that does not exceed a maximum of $\approx 50 \mu s$ (see the inset in Figure $6 \mathrm{~b}$ ). Theoretical estimate based on the scaling law above for collapse of the unfolded state of protein $\mathrm{L}(N=64)$ a maximum of $\tau_{c} \sim 4 \mu s$. Based on reconfiguration time measurements using Fluorescence Correlation Spectroscopy ${ }^{57,58}$ indicate that $\tau_{c}$ for small proteins could be less by an order of magnitude compared to the theoretical estimate. A larger value of $20 \mu s$, much shorter than the folding time, for reconfiguration time 
has also been reported for protein $\mathrm{L}^{59}$. For the larger DHFR $(N=154)$ there is nearly a $23 \%$ reduction in $R_{g}^{U}$ in $\sim 300 \mu s^{60}$, which is also considerably shorter than the folding time. All the studies show that collapse of the unfolded state, which increases with $N$, is on the order of at most tens of $\mu s$. Thus, we conclude that the time resolution in the most recent SAXS experiments on protein $\mathrm{L}(4 \mathrm{~ms})^{61}$, which is comparable to the folding time, is too long to shed light on compaction of the polypeptide chain. The presence of 15 His tags in the first study ${ }^{62}$ makes it difficult to ascertain the relevance for protein L. Indeed, the inability to accurately determine determine the characteristics of the unfolded state had also lead to erroneous conclusions about equilibrium collapse and kinetic foldability ${ }^{63}$, which has been corrected recently using smFRET experiments recently ${ }^{64}$.

Fast mixing experiments that simultaneously detect compaction and acquisition of structure on a number of proteins ${ }^{65-67}$ have produced ample evidence that collapse is an integral process of the folding process, as predicted by theory. Although it is likely that the simplified analysis of smFRET measurements overestimates the extent of collapse ${ }^{68}$, fast mixing SAXS experiments on a variety of proteins leave no doubt that the unfolded state is indeed more compact (albeit by only about $\approx 9 \%$ ) under native conditions despite persistent claims to the contrary based on SAXS data based largely on one protein (protein L) with large errors. It is a pity that the erroneous conclusion has resulted in unnecessary obfuscation. What is needed are high precision data for single domain proteins spanning a range of $N$ (say between 50-250), which cannot be easily obtained by SAXS alone ${ }^{69}$ but is more readily available in smFRET experiments.

Detection of $I_{E Q}$ in PDZ2 in single molecule pulling experiments: The downward curvature in the unfolding rate as a function $[C]$ in PDZ2 implies that an intermediate is populated $^{27}$. Single molecule pulling experiments are well suited to explore this finding more readily. Based on the free energy profile computed here and postulated elsewhere ${ }^{31}$, we suggest that unfolding by mechanical force $(f)$ would give rise to downward curvature in a plot of $\log k_{u}(f)$ versus $f$. At low forces the inner barrier separating the $N B A$ and $I_{E Q}$ would dominate whereas at high forces the second outer barrier is relevant for mechanical unfolding. The two sequential barrier picture implies that there would be two transition state distances with a switch between the two occurring as $f$ is increased. A plausible support for this argument comes from the observation that the Tanford $\beta_{T}$ as a function of $[C]$ exhibits a sigmoidal behavior (see Figure $4 \mathrm{in}^{31}$ ) with $\beta_{T}$ changing from 0.35 at low $[C]$ to 0.85 at high $[C]$. The 
scenario postulated here is distinct from that in SH3 domain in $\left[\log k_{u}(f), f\right]$ plot exhibits an upward curvature, which is a signature of parallel unfolding pathways with a switch between the transition state ensembles ${ }^{70}$ as opposed to the predicted sequential barrier model for PDZ2. Laser optical tweezer experiments are ideally suited to test our prediction.

Structures of the TSEs: It is interesting to compare the structures of TSE1 and TSE2 obtained in this work with those reported earlier ${ }^{29}$. The structure of TSE1 (Figure 5) show interactions involving $\beta_{2}, \beta_{3}$, and formation of the two helices. This is in sharp contrast to the conclusion in $^{29}$ suggesting that in TSE1 $\beta$ strands $1-6-4$ are structured whereas the other secondary structural elements are essentially disordered. Although the agreement between the predicted structures in our work and those reported $\mathrm{in}^{29}$ in TSE2 is better in that we find that it is native-like, there are crucial differences as well. In particular, the lack of stabilizing interactions involving $\beta_{1}$ and $\beta_{6}$ found in our simulations disagrees with the inferences drawn from the all atom molecular dynamics simulations using measured $\Phi$ values as constraints ${ }^{29}$. The differences are likely to be related to the completely different approaches used in the two studies. $\mathrm{In}^{29}$ the measured $\Phi$ values were used as constraints in standard all atom molecular dynamics simulations, which use inaccurate force fields. The procedure, while interesting, is not systematic in the sense the accumulation of errors both from the $\Phi$ values as well as the MD simulations is nearly impossible to quantify. More importantly, the putative TSE structures were not used to obtain the $P_{\text {fold }}$ values in the earlier study ${ }^{29}$, which casts doubt on whether the identified TSEs accurately represent the actual TSEs. We believe additional experiments, including perhaps double mutant cycles, would be needed to ascertain the nature of the TSEs in PDZ2.

\section{Concluding Remarks:}

We have used a phenomenological theory based on the MTM to simulate the folding of PDZ2 domain as function of temperature and urea. In addition to providing support to the folding mechanism discovered in experiments ${ }^{27}$ we have made a few testable predictions. (1) We have obtained a precise dependence of the melting point as a function of urea concentration, which can tested using standard calorimetry experiments. (2) The presence of high energy intermediate in the absence of added salt can be characterized using single molecule pulling experiments as 
shown using simulations for srcSH3 domain ${ }^{71}$ demonstrating that the excited state is sparsely $(O \approx 2-5) \%$ populated, which coincided with the findings based on NMR experiments ${ }^{72}$.

\section{Acknowledgements}

We are grateful to Ben Schuler and Gilad Haran for their interest and useful comments. This work was supported in part by a grant from the National Science Foundation to DT through CHE 13-61946. Z.L. acknowledges partial financial support from the National Natural Science Foundation of China (11104015) and the Fundamental Research Funds for the Central Universities (2012LYB08). 
[1] O’Brien, E. P.; Ziv, G.; Haran, G.; Brooks, B. R.; Thirumalai, D. P. Natl. Acad. Sci. USA, 2008, 105, 13403-13408.

[2] Liu, Z. X.; Reddy, G.; Thirumalai, D. J. Phys. Chem. B, 2012, 116, 6707-6716.

[3] Reddy, G.; Liu, Z. X.; Thirumalai, D. Proc. Natl. Acad. Sci. USA, 2012, 109, 17832 -17838.

[4] Reddy, G.; Thirumalai, D. J. Phys. Chem. B, 2015, 119, 11358-11370.

[5] O'Brien, E. P.; Brooks, B. R.; Thirumalai, D. J. Am. Chem. Soc., 2012, 134, 979-987.

[6] Chen, T.; Chan, H. S. Phys. Chem. Chem. Phys., 2014, 16(14), 6460-6479.

[7] Liu, Z. X.; Reddy, G.; O’Brien, E. P.; Thirumalai, D. Proc. Natl. Acad. Sci. USA, 2011, 108, $7787-7792$.

[8] Tozzini, V. Curr. Opin. Struct. Biol., 2005, 15, 144-150.

[9] Hyeon, C.; Thirumalai, D. Nat. Commun., 2011, 2:48\%.

[10] Elber, R.; Cardenas, A. E. in Comprehensive Biophysics, VOL 9: Simulation and Modeling, Egelman, EH, Ed., pp 2-26. 2011.

[11] Whitford, P. C.; Sanbonmatsu, K. Y.; Onuchic, J. N. Rep. Prog. Phys., 2012, 75, 076601.

[12] Thirumalai, D.; Liu, Z.; O’Brien, E. P.; Reddy, G. Curr. Opin. Struct. Biol., 2013, 23, 22-29.

[13] Kim, P. S.; Baldwin, R. L. Annu. Rev. Biochem., 1990, 59, 631-660.

[14] Wolynes, P. G.; Onuchic, J. N.; Thirumalai, D. Science, 1995, 267, 1619-1620.

[15] Dill, K. A.; Chan, H. S. Nature Struct. Biol., 1997, 4, 10-19.

[16] Daggett, V.; Fersht, A. R. Trends Biochem.Sci., 2003, 28, 18-25.

[17] Onuchic, J. N.; Wolynes, P. G. Curr. Opin. Struct. Biol., 2004, 14, 70-75.

[18] Dill, K. A.; Ozkan, S. B.; Shell, M. S.; Weikl, T. R. Annu. Rev. Biophys., 2008, 37, 289-316.

[19] Schuler, B.; Eaton, W. A. Curr. Opin. Struct. Biol., 2008, 18, 16-26.

[20] Shakhnovich, E. Chem. Rev., 2006, 106, 1559-1588.

[21] Thirumalai, D.; O'Brien, E. P.; Morrison, G.; Hyeon, C. Annu. Rev. Biophys., 2010, 39, 159-183.

[22] Dill, K. A.; MacCallum, J. L. Science, 2012, 338, 1042-1046.

[23] Piana, S.; Klepeis, J. L.; Shaw, D. E. Curr. Opin. Struct. Biol., 2014, 24, 98-105.

[24] Harris, B. Z.; Lim, W. A. J. Cell Sci., 2001, 114, 3219-3231.

[25] Kim, E. J.; Sheng, M. Nat. Rev. Neurosci., 2004, 5, 771-781. 
[26] Jemth, P.; Gianni, S. Biochemistry, 2007, 46, 8701-8708.

[27] Gianni, S.; Calosci, N.; Aelen, J. M. A.; Vuister, G. W.; Brunori, M.; Travaglini-Allocatelli, C. Protein Eng. Des. Sel., 2005, 18, 389-395.

[28] Sicorello, A.; Torrassa, S.; Soldi, G.; Gianni, S.; Travaglini-Allocatelli, C.; Taddei, N.; Relini, A.; Chiti, F. Biophys. J., 2009, 96, 2289-2298.

[29] Gianni, S.; Geierhaas, C. D.; Calosci, N.; Jemth, P.; Vuister, G. W.; Travaglini-Allocatelli, C.; Vendruscolo, M.; Brunori, M. Proc. Natl. Acad. Sci. USA, 2007, 104, 128-133.

[30] Pincus, D. L.; Cho, S. S.; Hyeon, C. B.; Thirumalai, D. Prog. Mol. Biol. Transl. Sci., 2008, 84, $203-250$.

[31] Ivarsson, Y.; Travaglini-Allocatelli, C.; Jemth, P.; Malatesta, F.; Brunori, M.; Gianni, S. J. Biol. Chem., 2007, 282, 8568-8572.

[32] Hyeon, C.; Dima, R. I.; Thirumalai, D. Structure, 2006, 14, 1633-1645.

[33] Camacho, C. J.; Thirumalai, D. Proteins: Structure, Function and Genetics, 1995, 22, 28-40.

[34] Klimov, D. K.; Thirumalai, D. Proteins - Struct. Funct. Gene., 2001, 43, 465-475.

[35] Gin, B. C.; Garrahan, J. P.; Geissler, P. L. J. Mol. Biol., 2009, 392(5), 1303-1314.

[36] Best, R. B.; Hummer, G.; Eaton, W. A. Proc. Natl. Acad. Sci., 2013, 110(44), 17874-17879.

[37] Betancourt, M.; Thirumalai, D. Prot. Sci., 1999, 8, 361-369.

[38] Dima, R. I.; Thirumalai, D. J. Phys. Chem. B, 2004, 108, 6564-6570.

[39] Veitshans, T.; Klimov, D.; Thirumalai, D. Fold. Des., 1997, 2, 1-22.

[40] Hansmann, U.; Okamoto, Y.; Eisenmenger, F. Chem. Phys. Lett., 1996, 259, 321-330.

[41] Okamoto, Y.; Hansmann, U. J. Phys. Chem., 1995, 99, 11276-11287.

[42] Zhou, R. H.; Berne, B. J.; Germain, R. P. Natl. Acad. Sci. USA, 2001, 98, 14931-14936.

[43] Sugita, Y.; Okamoto, Y. Chem. Phys. Lett., 1999, 314, 141-151.

[44] Honeycutt, J. D.; Thirumalai, D. Biopolymers, 1992, 32, 695-709.

[45] Ermak, D. L.; McCammon, J. A. J. Chem. Phys., 1978, 69, 1352-1369.

[46] Camacho, C. J.; Thirumalai, D. Proc. Natl. Acad. Sci. USA, 1993, 90, 6369-6372.

[47] Ivarsson, Y.; Travaglini-Allocatelli, C.; Morea, V.; Brunori, M.; Gianni, S. Protein Eng. Des. Sel., 2008, 21, 155-160.

[48] Du, R.; Pande, V. S.; Grosberg, A. Y.; Tanaka, T.; Shakhnovich, E. I. J. Chem. Phys., 1998, 108,334 . 
[49] Camacho, C. J.; Thirumalai, D. Phys. Rev. Lett., 1993, 71(15), 2505-2508.

[50] Garcia-Manyes, S.; Dougan, L.; Badilla, C.; Brujic, J.; J. M. Fernandez, J. Proc. Natl. Acad. Sci., 2009, 106, 10534-10539.

[51] Ziv, G.; Haran, G. J. Am. Chem. Soc., 2009, 131, 2942-2947.

[52] Ziv, G.; Thirumalai, D.; Haran, G. Phys. Chem. Chem. Phys., 2009, 11, 83-93.

[53] Haran, G. Curr. Opin. Struct. Biol., 2012, 22, 14-20.

[54] de Gennes, P. G. J. Phys. Lett., 1985, 46, L639-L642.

[55] Pitard, E.; Orland, H. Europhys. Lett., 1998, 41, 467-472.

[56] Thirumalai, D. J. Phys. I (Fr.), 1995, 5, 1457-1467.

[57] Nettels, D.; Gopich, I. V.; Hoffmann, A.; Schuler, B. Proc. Natl. acad. Sci., 2007, 104, 2655-2660.

[58] Soranno, A.; Buchli, B.; Nettels, D.; Cheng, R.; Mueller-Spaeth, S.; Pfeil, S. H.; Hoffmann, A.; Lipman, E. A.; Makarov, D. E.; Schuler, B. Proc. Natl. acad. Sci., 2012, 109, 17800-17806.

[59] Waldauer, S. A.; Bakajin, O.; Lapidus, L. J. Proc. Natl. acad. Sci., 2010, 107, 13713-13717.

[60] Arai, M.; Kondrashkina, E.; Kayatekin, C.; Matthews, C. R.; Iwakura, M.; Bilsel, O. J. Mol. Biol., 2007, 368, 219-229.

[61] Yoo, T. Y.; Meisburger, S. P.; Hinshaw, J.; Pollack, L.; Haran, G.; Sosnick, T. R.; Plaxco, K. J. Mol. Biol., 2012, 418, 226-236.

[62] Plaxco, K.; Millett, I.; Segel, D.; Doniach, S.; Baker, D. Nat. Struct. Biol., 1999, 6, 554-556.

[63] Millet, I.; Townsley, L.; Chiti, F.; Doniach, S.; Plaxco, K. Biochemistry, 2002, 41, 321-325.

[64] Hofmann, H.; Soranno, A.; Borgia, A.; Gast, K.; Nettels, D.; Schuler, B. Proc. Natl. Acad. Sci., 2012, 109, 16155-16160.

[65] Chan, C.; Hu, Y.; Takahashi, S.; Rousseau, D.; Eaton, W.; Hofrichter, J. Proc. Natl. Acad. Sci., 1997, 94, 1779-1784.

[66] Akiyama, S.; Takahashi, S.; Kimura, T.; Ishimori, K.; Morishima, I.; Nishikawa, Y.; Fujisawa, T. Proc. Natl. Acad. Sci., 2002, 99, 1329-1334.

[67] Kimura, T.; Uzawa, T.; Ishimori, K.; Morishima, I.; Takahashi, S.; Konno, T.; Akiyama, S.; Fujisawa, T. Proc. Natl. Acad. Sci., 2005, 102, 2748-2753.

[68] O’Brien, E. P.; Morrison, G.; Brooks, B. R.; Thirumalai, D. J. Chem. Phys., 2009, 130, 124903.

[69] Trewhella, J.; Hendrickson, W. A.; Kleywegt, G. J.; Sali, A.; Sato, M.; Schwede, T.; Svergun, D. I.; Tainer, J. A.; Westbrook, J.; Berman, H. M. Structure, 2013, 21, 875-881. 
bioRxiv preprint doi: https://doi.org/10.1101/037283; this version posted January 22, 2016. The copyright holder for this preprint (which was not certified by peer review) is the author/funder, who has granted bioRxiv a license to display the preprint in perpetuity. It is made available under aCC-BY-NC-ND 4.0 International license.

[70] Guinn, E. J.; Jagannathan, B.; Marqusee, S. Nat. Comm., 2015, 6, 6861.

[71] Zhuravlev, P. I.; Reddy, G.; Straub, J. E.; Thirumalai, D. J. Mol. Biol., 2014, 426, 2653-2666.

[72] Neudecker, P.; Robustelli, P.; Cavalli, A.; Walsh, P.; Lundstrom, P.; Zarrine-Afsar, A.; Sharpe, S.; Vendruscolo, M.; Kay, L. E. Science, 2012, 336, 362-366. 


\section{Figure Captions}

Figure 1: Thermodynamics of folding. (a) Ribbon diagram representation of PDZ2(PDB code: 1GM1). (b) Temperature dependence of specific heat (blue) and total energy (magenta). (c) Free energy profile at $T_{m}$ as a function of $\chi$. The values $\chi_{c}^{N}$ and $\chi_{c}^{D}$ are used to classify the major equilibrium states. The shaded areas give putative regions for the two transition state ensembles. (d) Fraction of molecules in NBA(black), UBA(red) and IBA(green) as functions of temperature.

Figure 2: Denaturation effects. (a) Fraction of molecules in the NBA (black), UBA (red), and IBA (green) as a function of urea concentration $[C]$. For comparison, the experimental curve for $f_{N B A}[C]$ (blue) is shown. (b) $[C]$ dependence of free energy of stability of the native state with respect to the unfolded state. Fit to a linear function yields $\Delta G_{N U}=\Delta G_{N U}(0)+m[C]$ where $\Delta G_{N U}(0)=-3.09 \mathrm{kcal} / \mathrm{mol}$ and $m=1.35 \mathrm{kcal} / \mathrm{mol} . M$. (c) Heat capacity versus temperature for different values of $[C]$. (d) The $[C]$ dependence of the melting temperature. The line is a fit to $T_{m}[C]=T_{m}(0)-B[C]$ where $T_{m}(0)=324 \mathrm{~K}$ and $B=-3.1 K M^{-1}$.

Figure 3: Equilibrium collapse. (a) Average $<R_{g}>$ (black) as a function of $[C]$. Red, green and blue curves correspond to $<R_{g}>$ of the folded, unfolded and $I_{E Q}$ states, respectively. The scale for the unfolded state is on the right. (b)Distribution $P\left(R_{g}\right)$ of $R_{g}$ for various concentrations of urea. The inset shows $P\left(R_{g}\right)$ for $[C]=0$ (black), $[C]=2.3$ (blue), and $[C]=5.0$ (orange) corresponding to the extended conformations $\left(R_{g}>16 \AA\right.$ ). (c) Distance distribution function $P(r)$, the inverse Fourier transform of the scattering intensity, for 0M (black), 1.0M (red), 2.3M (green), and 5.0M (blue) urea. Here, $r$ is the distance between all non-covalently linked beads.

Figure 4: Free energy profiles versus $\chi$ at different $[C]$. (a) $T=T_{m}$. (b) $T=T_{s}$. The values of $[C]$ measured in $\mathrm{M}$ from top to bottom are $0,1,2,2.3,3,4,5,6,7$, and 8.

Figure 5: Quantifying the transition state ensembles. (a) Distribution $P\left(\Delta_{R}\right)$, of the $\Delta_{R}=\left(\Delta_{U}-\Delta_{T S E}\right) /\left(\Delta_{U}-\Delta_{N}\right)$, which is the fraction of buried solvent accessible surface area 
relative to the unfolded structures. The average $<\Delta_{R}>=0.23$ for TSE1 and $<\Delta_{R}>=0.68$ for TSE2. These values coincide qualitatively with Tanford $\beta$ parameters extracted from the observed Chevron plot. A few of the TSE1 (TSE2) structures are displayed on the left (right) respectively. (b)The distributions of $\chi$ computed from 400 simulation trajectories spawned from the transition state structures in TSE1. Data are shown for the four different structures. The distribution shows that roughly half of these trajectories go to the folded basin of attraction $\left(P_{\text {fold }} \approx 0.5\right)$.(c) Contact map of the native state ensemble (upper left) and the one for the TSE1(lower right). The scale on the right gives the probability of contact formation. (d) and (e) Same as (b) and (c), respectively except the results are for TSE2.

Figure 6: Folding and collapse kinetics. (a) Fraction of molecules that have not folded $([C]=0.0 M)$ as a function of time. The line is an exponential fit $\left(P_{u}(t)=e^{-} t / \tau_{F}\right.$ with $\tau_{F}=5.8 \mathrm{~ms}$ ) to the data. (b) Collapse kinetics monitored by the time-dependent average $<R_{g}(t)>$ as a function of $t$ with the line giving an exponential fit to the data. The collapse time of PDZ2 is $\tau_{c}=4.1 \mathrm{~ms}$.

Figure 7: Two major folding pathways monitored by $\chi$ as a function of $t$. (a) and (b) show two representative trajectories showing that the native state is reached by sampling both $I_{1}$ and $I_{2}$. (c) and (d) show two folding trajectories in which the polypeptide chain samples only $I_{1}$, often multiple times, before reaching the folded state. (e) Structures for I1 and I2.

Figure 8: Comparing $I_{E Q}, I_{1}$, and $I_{2}$. (a)Average fraction of native contacts formed for residues, $f_{Q}$, for $I_{E Q}$ (black) and $I_{1}$ (red). (b) $f_{Q}$ for $I_{E Q}$ (black) and $I_{2}$ (blue). (c) Correlation between $f_{Q}$ f for $I_{E Q}$ and $I_{1}$. The correlation coefficient is near unity. (d) Relation between $f_{Q^{S}}$ for $I_{E Q}$ and $I_{2}$ with correlation coefficient $\approx 0.9$. (e) Structures of the three intermediates.

Figure 9: Table of contents. 
(a)

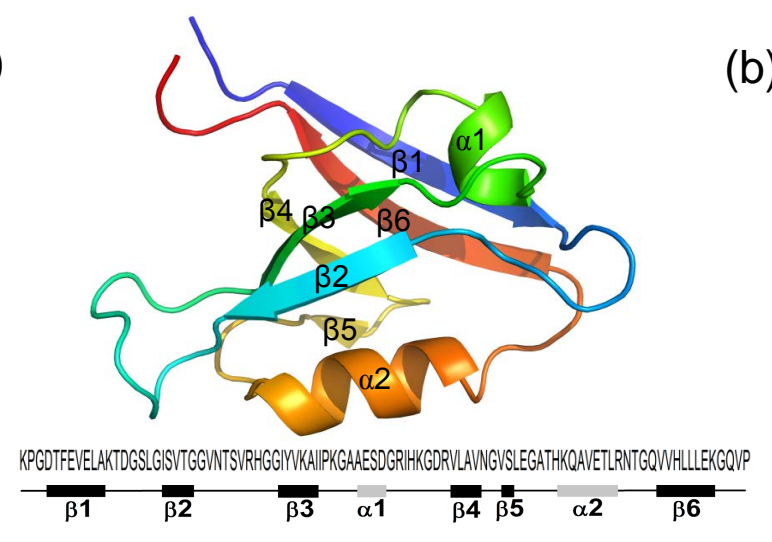

(c)

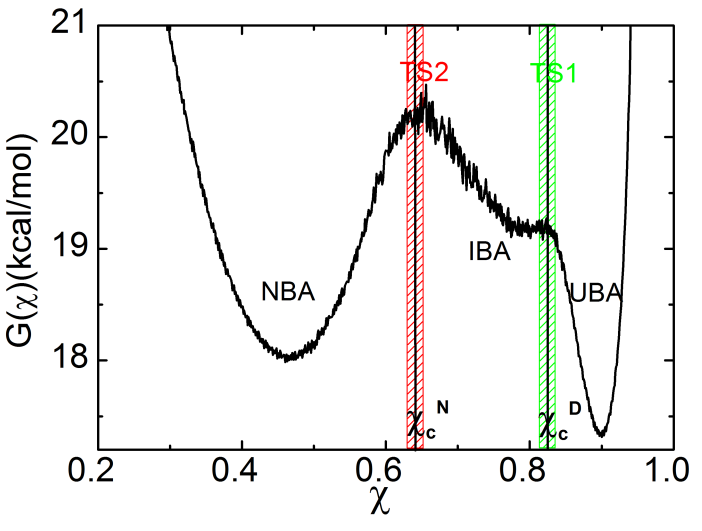

(b)

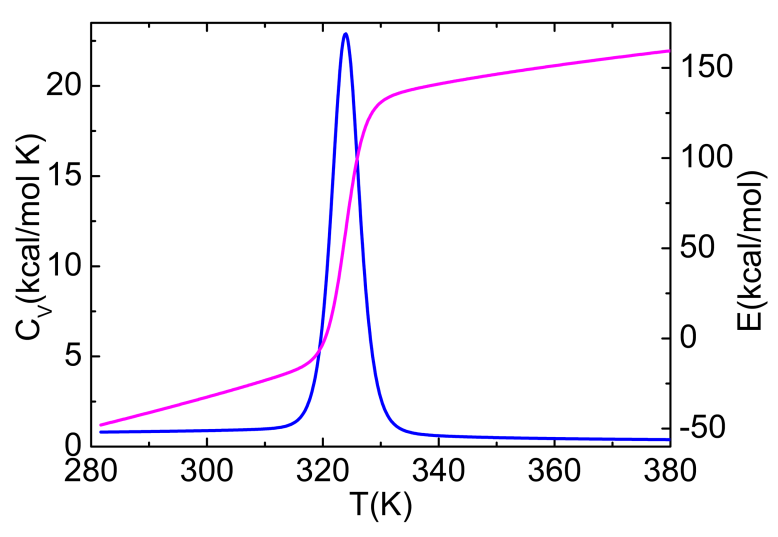

(d)

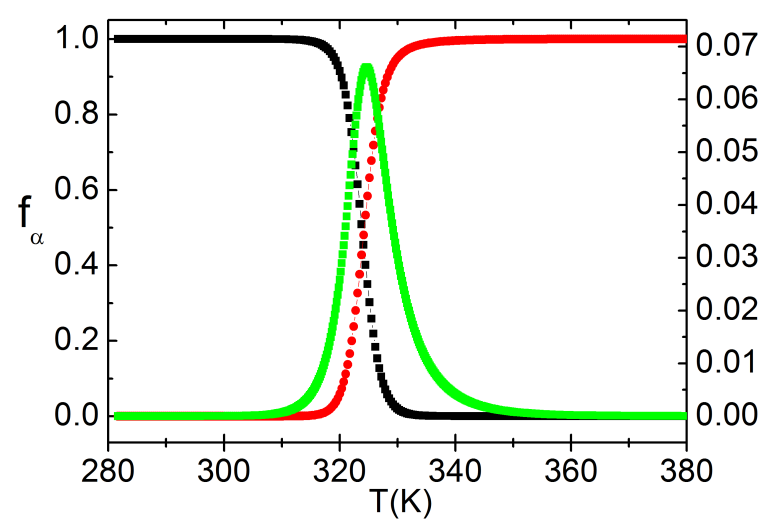

FIG. 1: 

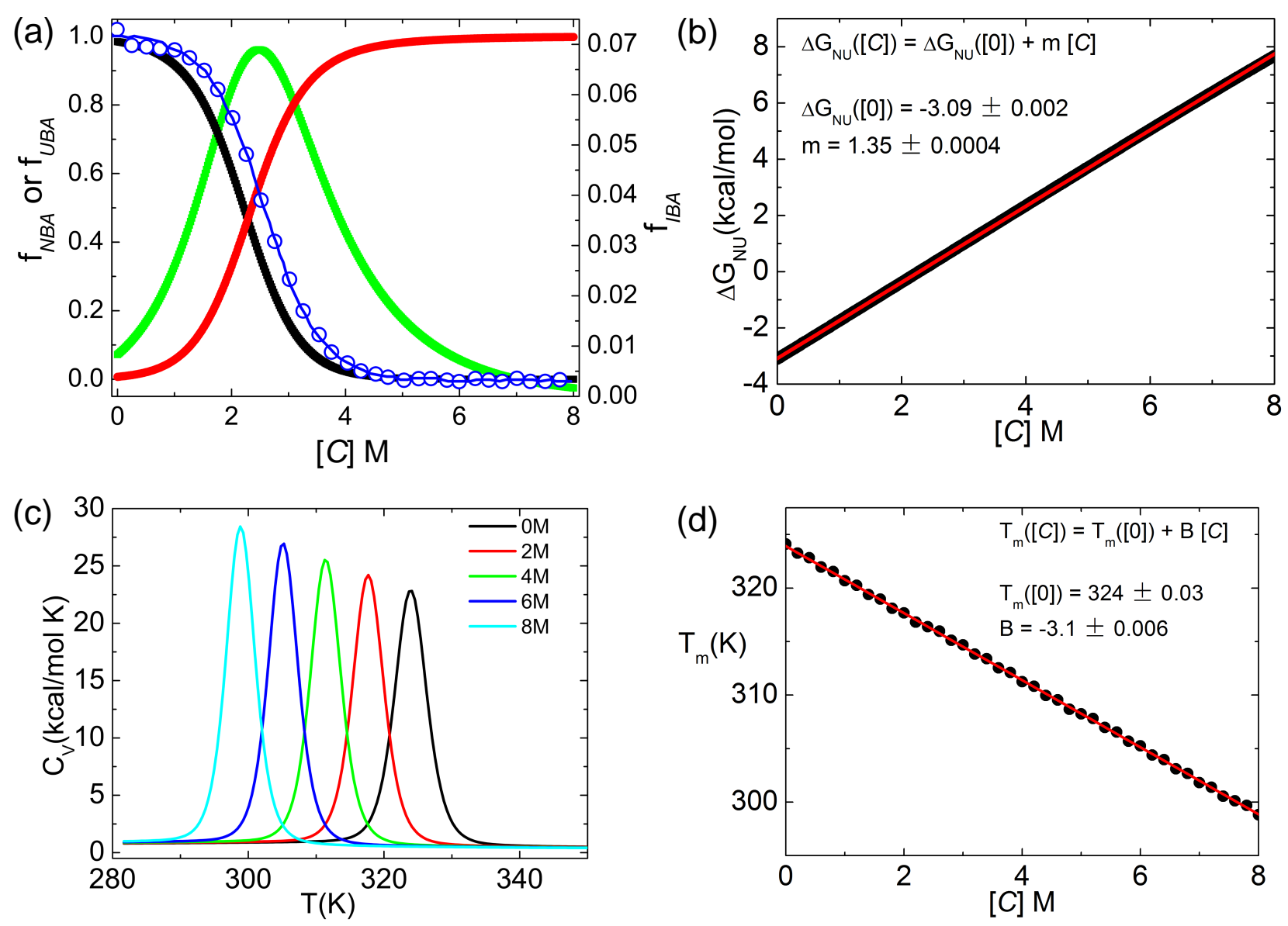

FIG. 2: 
bioRxiv preprint doi: https://doi.org/10.1101/037283; this version posted January 22, 2016. The copyright holder for this preprint (which was not certified by peer review) is the author/funder, who has granted bioRxiv a license to display the preprint in perpetuity. It is made available under aCC-BY-NC-ND 4.0 International license.

(a)

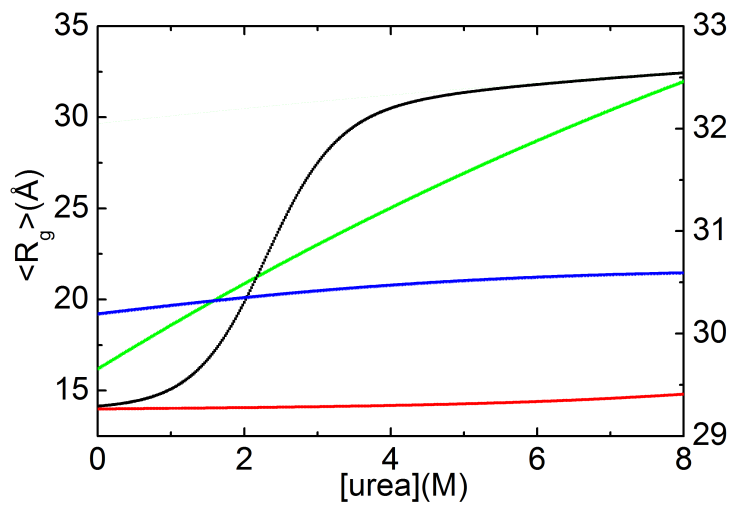

(b)

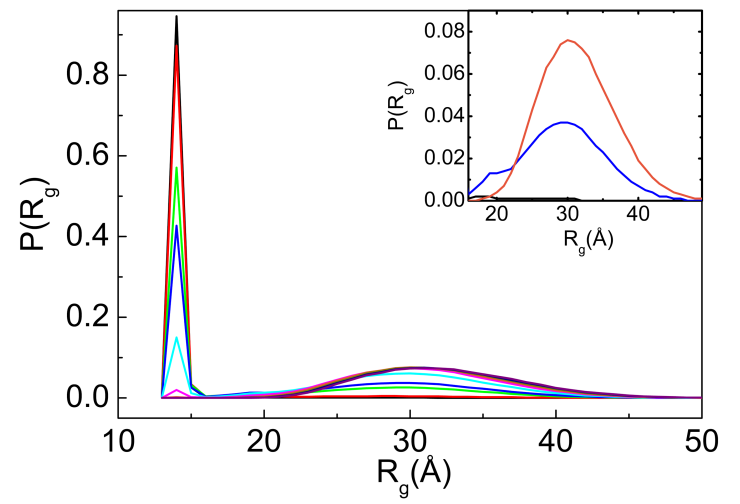

(c)

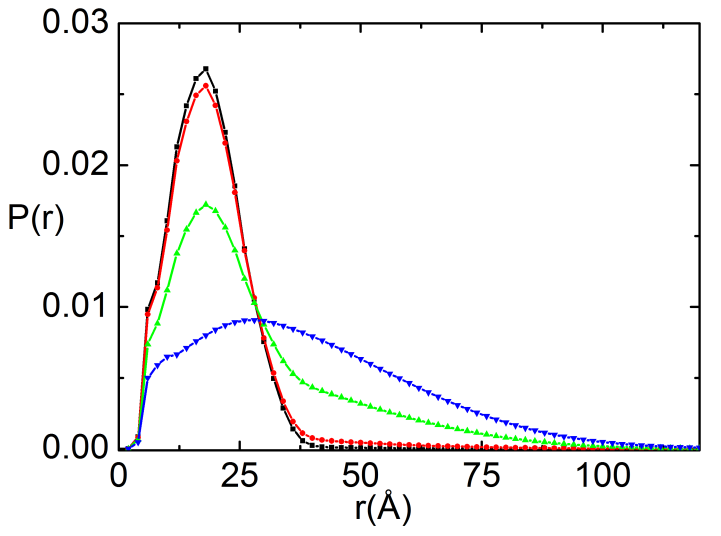

FIG. 3: 
bioRxiv preprint doi: https://doi.org/10.1101/037283; this version posted January 22, 2016. The copyright holder for this preprint (which was not certified by peer review) is the author/funder, who has granted bioRxiv a license to display the preprint in perpetuity. It is made available under aCC-BY-NC-ND 4.0 International license.
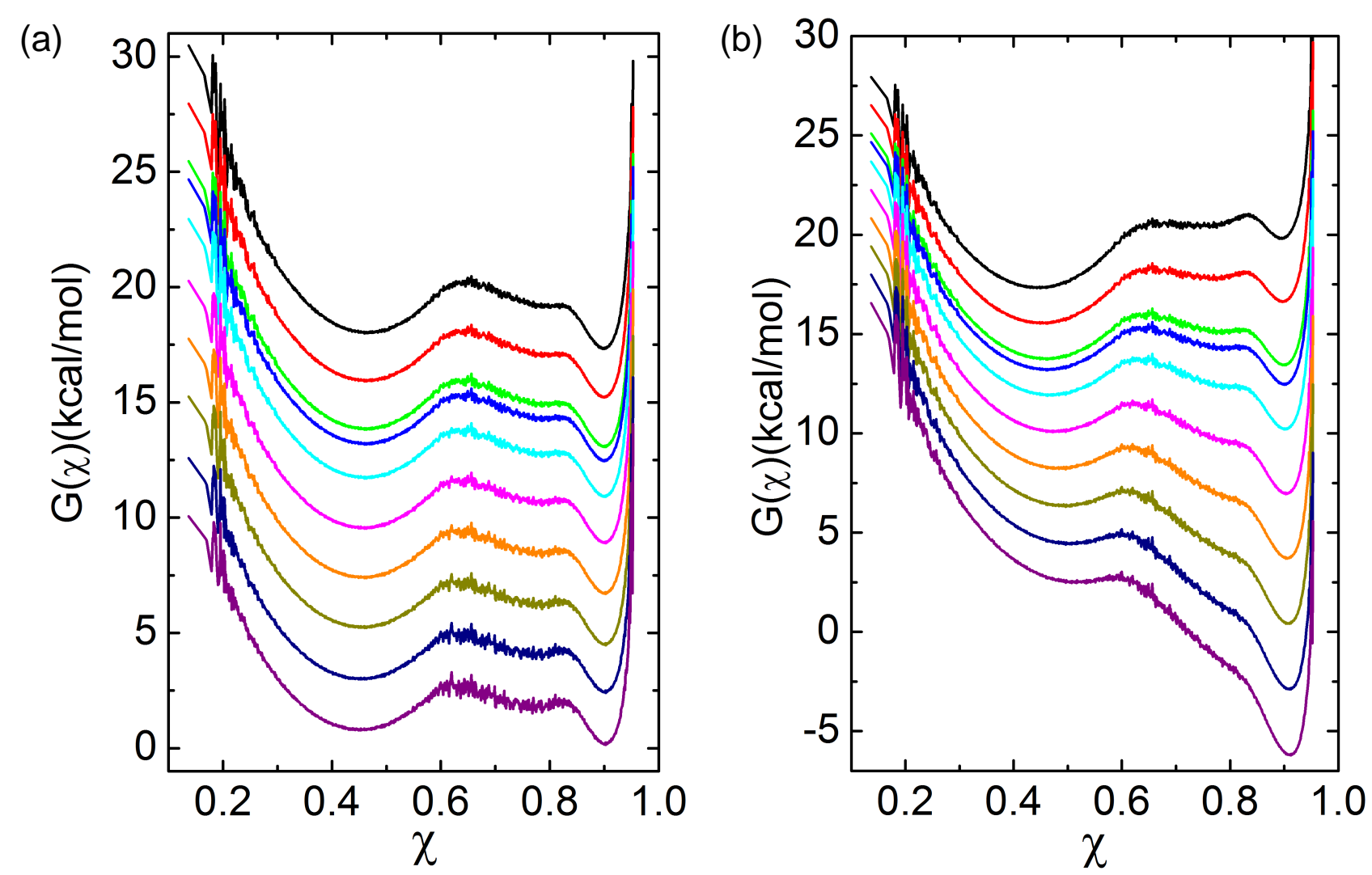

FIG. 4: 

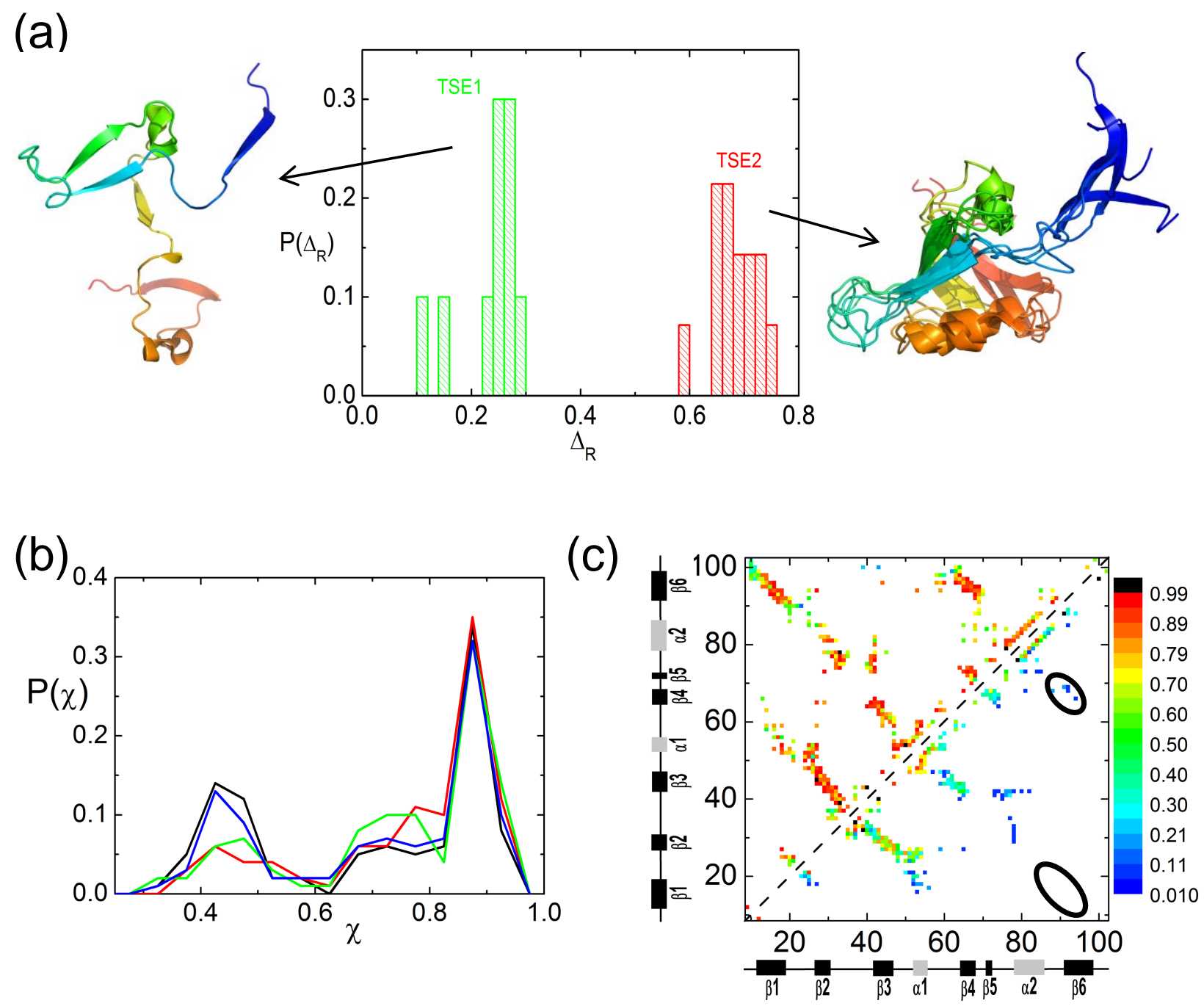

(d)

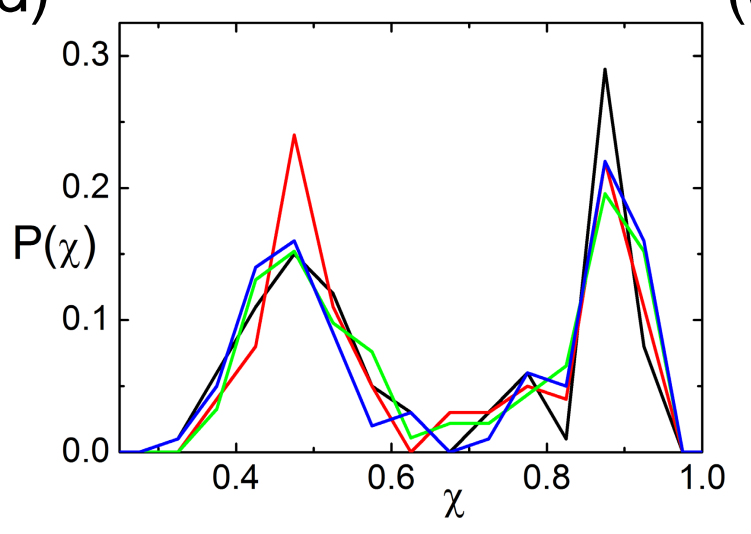

(e)

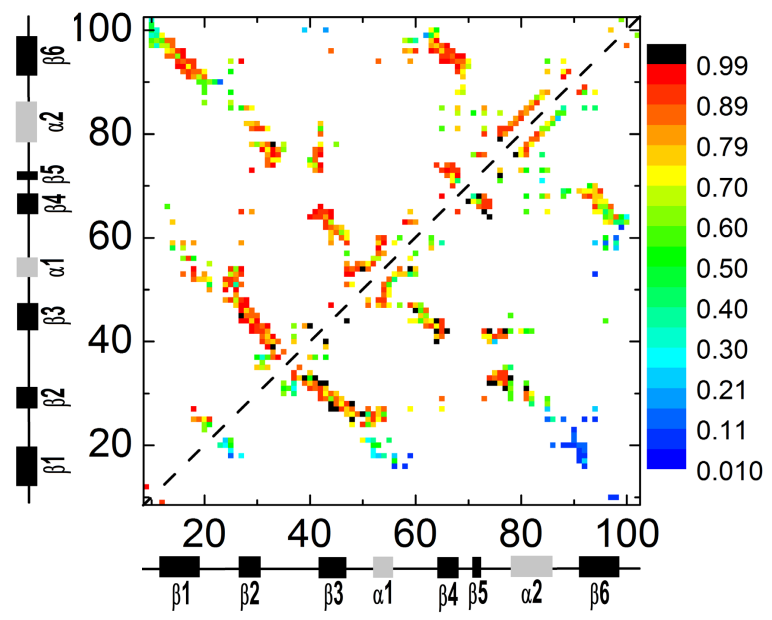

FIG. 5: 
bioRxiv preprint doi: https://doi.org/10.1101/037283; this version posted January 22, 2016. The copyright holder for this preprint (which was not certified by peer review) is the author/funder, who has granted bioRxiv a license to display the preprint in perpetuity. It is made available under aCC-BY-NC-ND 4.0 International license.

(a)

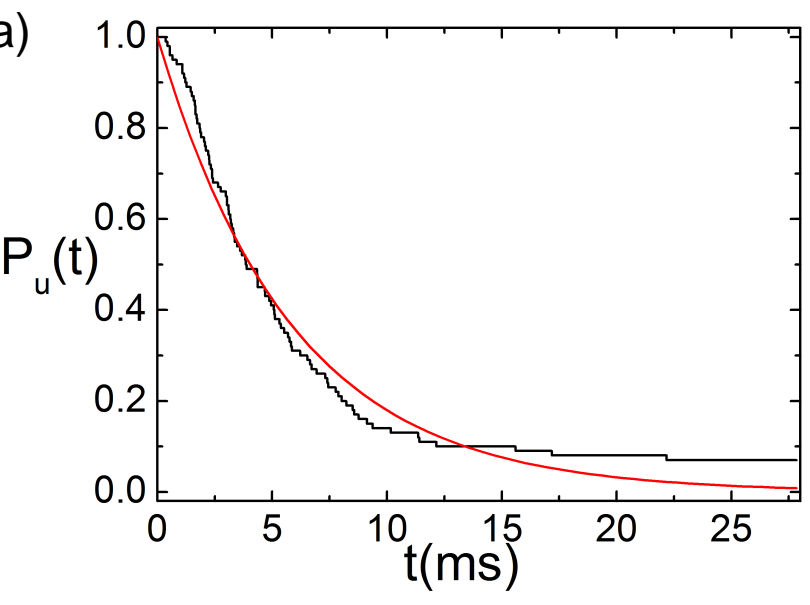

(b)

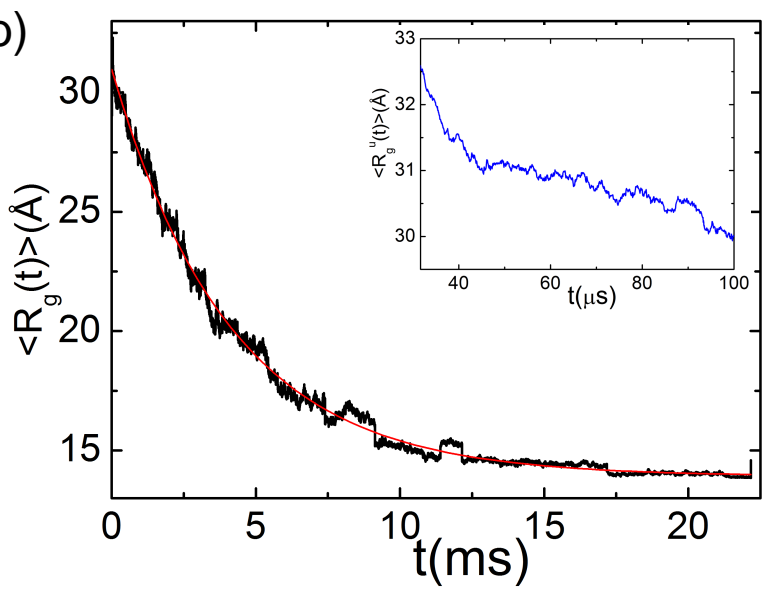

FIG. 6: 
bioRxiv preprint doi: https://doi.org/10.1101/037283; this version posted January 22, 2016. The copyright holder for this preprint (which was not certified by peer review) is the author/funder, who has granted bioRxiv a license to display the preprint in perpetuity. It is made available under aCC-BY-NC-ND 4.0 International license.
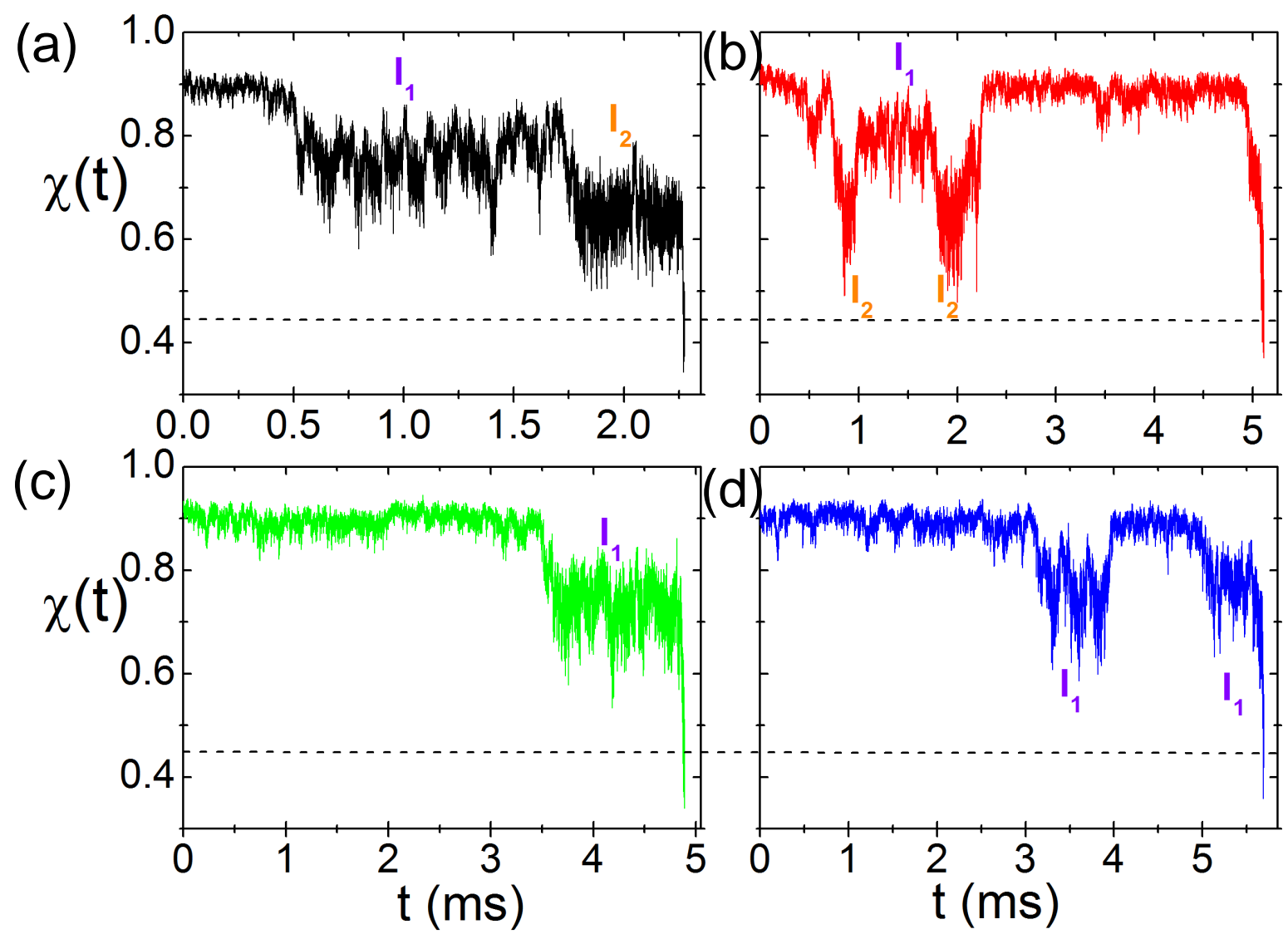

(c)

(e)
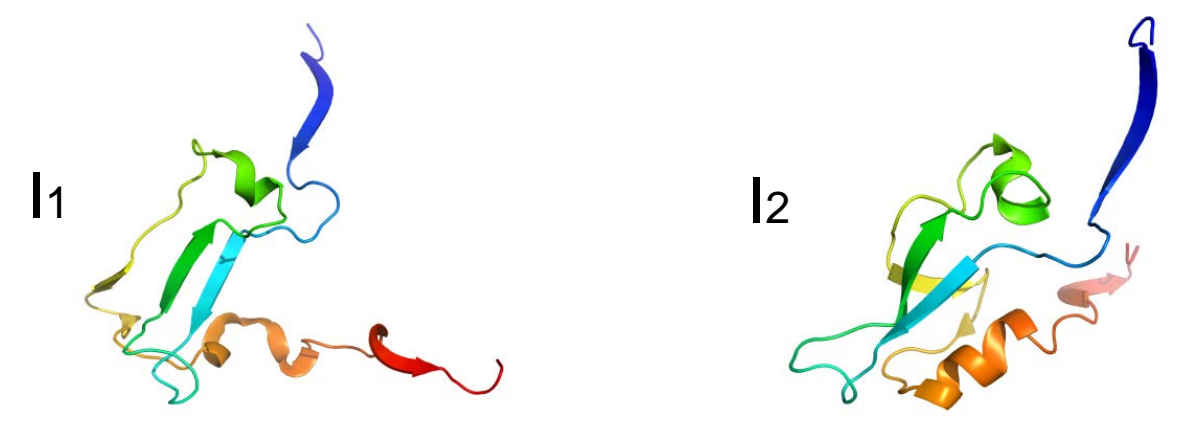

FIG. 7: 
(a)

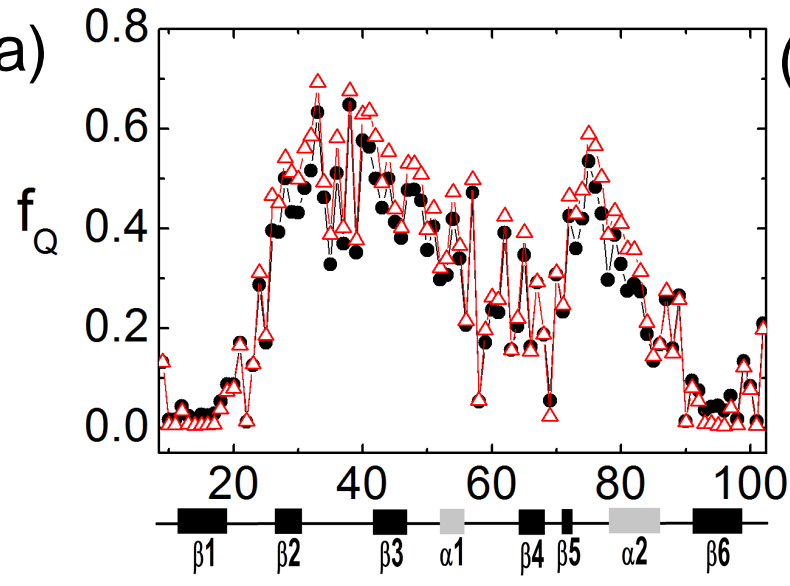

(c)
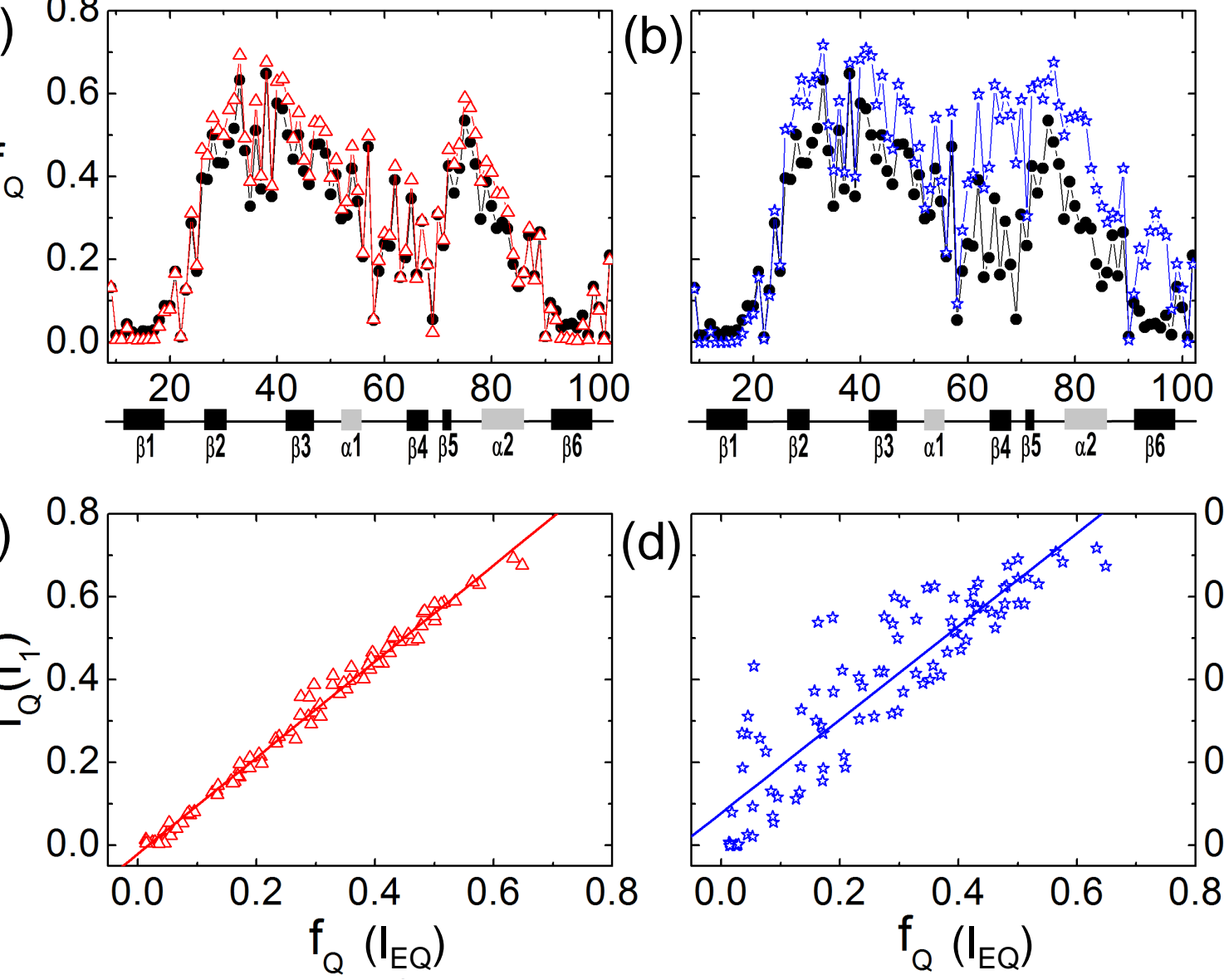

(e)
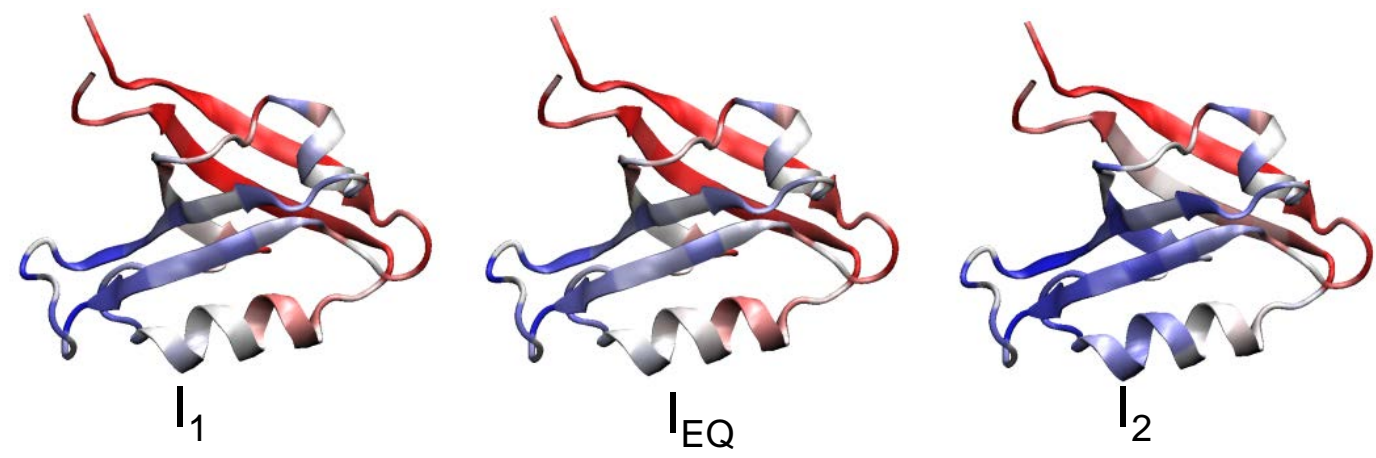

FIG. 8: 
bioRxiv preprint doi: https://doi.org/10.1101/037283; this version posted January 22, 2016. The copyright holder for this preprint (which was not certified by peer review) is the author/funder, who has granted bioRxiv a license to display the preprint in perpetuity. It is made available under aCC-BY-NC-ND 4.0 International license.

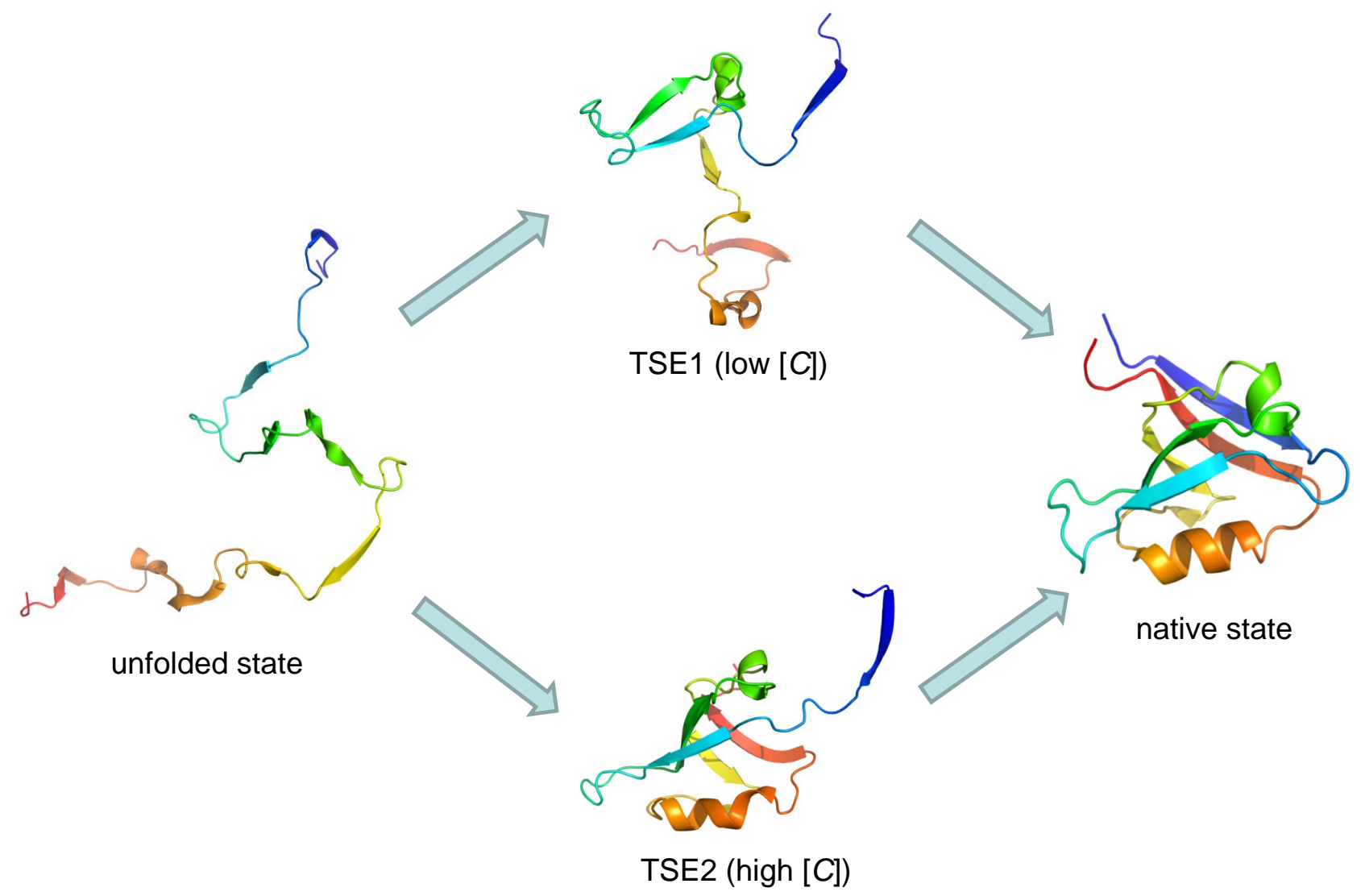

FIG. 9: 